\title{
PROPER OR WEAK EFFICIENCY VIA SADDLE POINT CONDITIONS IN CONE CONSTRAINED NONCONVEX VECTOR OPTIMIZATION PROBLEMS *
}

\author{
FABIÁN FLORES-BAZÁN ${ }^{\dagger}$, CRISTIÁN VERA , AND GIANDOMENICO MASTROENI $^{\S}$
}

\begin{abstract}
Motivated by many applications (for instance, some production models in finance require infinitydimensional commodity spaces, and the preference is defined in terms of an ordering cone having possibly emtpy interior), this paper deals with a unified model which involves preference relations that are not necessarily transitive or reflexive. Our study is carried out by means of saddle point conditions for the generalized Lagrangian associated with a cone constrained nonconvex vector optimization problem. We establish a necessary and sufficient condition for the existence of a saddle point in case the multiplier vector related to the objective function belongs to the quasi interior of the polar of the ordering set. Moreover, exploiting suitable Slater-type constraints qualifications involving the notion of quasi-relative interior, we obtain several results concerning the existence of a saddle point, which serve to get efficiency, weak efficiency and proper efficiency. Such results generalize to the nonconvex vector case existing conditions in the literature. As a byproduct, we propose a notion of proper efficient solution for a vector problem with explicit constraints. Applications to optimality conditions for vector optimization problems are provided with particular attention to bicriteria problems where optimality conditions for efficiency, proper efficiency and weak efficiency, are stated, both in a geometric form and by means of the level sets of the objective functions.
\end{abstract}

Key words. Quasi relative interior, saddle point, nonconvex vector optimization.

Mathematics subject classification 2000. Primary: 90C29; Secondary: 90C46, 49A52, 49B27, 90C31.

1. Introduction. Let $X, Y, Z$ be Hausdorff real locally convex topological vector spaces (t.v.s.), $Q \subseteq Z$ be a nonempty closed convex cone and $C$ be a nonempty subset of $X$. Given $f: X \rightarrow Y$ and $g: X \rightarrow Z$, we consider the following cone constrained vector optimization problem which consists in finding

$$
\bar{x} \in K: \quad f(x)-f(\bar{x}) \notin-S, \quad \forall x \in K, x \neq \bar{x},
$$

where $K:=\{x \in C: g(x) \in-Q\}$ and the ordering is given by the set $S \subset Y$, which defines not necessarily a transitive or reflexive preference relation. When a preference on $Y$ is defined by a convex cone $P \subseteq Y$, usually $S$ satisfies $S+P=S$; suitably choosing the set $S$, it is possible to recover the most common definitions of efficiency (see Section 3 ).

In this paper, exploiting the image space approach [18], we analyze conditions ensuring the existence of a saddle point for the generalized Lagrangian function associated with problem (1.1) (defined in (3.7)). Such conditions are obtained by means of the notion of quasi relative interior $[5,4]$, that recently has widely been considered in the literature $[10,6,13,16,32,33,35]$.

*THIS RESEARCH, FOR THE FIRST AUTHOR, WAS SUPPORTED IN PART BY CONICYT-CHILE THROUGH FONDECYT 112-0980 AND BASAL PROJECTS, CMM, UNIVERSIDAD DE CHILE.

${ }^{\dagger}$ Departamento de Ingeniería Matemática, $\mathrm{CI}^{2} \mathrm{MA}$, Facultad de Ciencias Físicas y Matemáticas, Universidad de Concepción, Casilla 160-C, Concepción, Chile. E-mail: fflores@ing-mat.udec.cl

¥ Facultad de Ingeniera y Arquitectura, Universidad Arturo Prat, Casilla 121, Iquique, Chile. E-mail: cristver@unap.cl

$\S$ Department of Computer Science, University of Pisa, L.go B. Pontecorvo, 3, 56127 Pisa, Italy. E-mail: gmastroeni@di.unipi.it 
This allows us to consider problem (1.1) where the set $S$ and the cone $Q$ may have empty topological interior (see e.g., $[5,22,11,7])$. Indeed, in the literature the analysis of saddle point conditions for vector optimization problems is in general carried out under the assumptions that the ordering set is a cone with nonempty interior as well as the cone $Q$ appearing in the constraints $[2,20,29]$. Our results extend to the vector case some analogous theorems proved for a scalar cone constrained extremum problem as considered in $[8,6,19,16]$.

Some concrete models in infinite-dimensional spaces may be found in [24]; whereas for a theoretical treatment of vector optimization problems concerning existence and optimality conditions, we refer $[28,24]$.

We recall that every nonempty convex subset of a separable Banach space admits quasirelative interior points [3, Theorem 2.19]; and there are only a few infinite-dimensional spaces whose natural ordering cones have nonempty interior: like $l^{\infty}$, the space of bounded variation on $\mathbb{R}$, or the space of continuous real-valued functions defined on a compact set of $\mathbb{R}^{n}$.

The paper is organized as follows. Section 2 provides the basic definitions, notations and preliminaries on quasi (relative) interior and separation theorems of convex sets. In Section 3 we state a characterization of a saddle point condition ensuring that the vector of multipliers associated with the objective function belongs to the interior of the polar of the ordering set; then we present the main results on the existence of a saddle point condition and we show that, under generalized Slater type contraints qualifications, the vector of multipliers associated with the objective function does not vanish; as a byproduct, we propose a notion of proper efficient point for vector optimization problems under explicit constraints. Applications to optimality conditions for efficiency or weak efficiency in vector optimization problems are considered in Section 4; in particular, we show that in the presence of suitable generalized convexity assumptions, the existence of a saddle point is a necessary optimality condition for a weak efficient point (or efficient point) of (1.1). In Section 5 we analyze more in details the case of a bicriteria problem with a geometric constraint set for which we point out that the convexity of the closure of the cone generated by the extended image set associated with the objective function turns out to be necessary and sufficient for the existence of a saddle point for the Lagrangian function, or equivalently in this case, for a scalarization of the given problem. Optimality conditions for such problems are provided both in a geometric form and by means of suitable properties of the level sets of the objective functions.

2. Preliminary results. Let us recall the basic notations and preliminary results that will be used throughout the paper.

A set $P \subseteq Y$ is said to be a cone if $t P \subseteq P, \forall t \geq 0$; given $A \subseteq Y, \operatorname{co}(A)$, int $A, \bar{A}$, denote the convex hull of $A$, the topological interior of $A$ and the closure of $A$, respectively. We define

$$
\operatorname{cone}(A):=\bigcup_{t \geq 0} t A, \quad \operatorname{cone}_{+}(A):=\bigcup_{t>0} t A .
$$

Evidently, cone $(A)=$ cone $_{+}(A) \cup\{0\}$ and therefore $\overline{\text { cone }(A)}=\overline{\operatorname{cone}}(A)=\overline{\text { cone }_{+}(A)}$.

We set $\mathbb{R}_{+}^{m}:=\left\{x \in \mathbb{R}^{m}: x \geq 0\right\}$.

Given a convex set $A \subseteq Y$ and $x \in A$, the normal cone to $A$ at $x$ is defined by $N_{A}(x):=$ $\left\{\xi \in Y^{*}:\langle\xi, a-x\rangle \leq 0, \quad \forall a \in A\right\}$. We say that $x \in A$ is a (see, e.g., [6]): 
(a) quasi interior point of $A$, denoted by $x \in$ qi $A$, if $\overline{\operatorname{cone}}(A-x)=Y$, or equivalently, $N_{A}(x)=\{0\}$

(b) quasi relative interior point of $A$, denoted by $x \in$ qri $A$, if $\overline{\operatorname{cone}}(A-x)$ is a linear subspace of $Y$, or equivalently, $N_{A}(x)$ is a linear subspace of $Y^{*}$.

For any convex set $A$, we have that $([27,6])$ qi $A \subseteq$ qri $A$ and, int $A \neq \emptyset \operatorname{implies}$ int $A=$ qi $A$. Similarly, if qi $A \neq \emptyset$, then qi $A=$ qri $A$. Moreover [5], if $Y$ is a finite dimensional space, then qi $A=\operatorname{int} A$ and qri $A=$ ri $A$, where ri $A$ denotes the relative interior of $A$, which is the interior with respect to the affine hull of $A$. For additional properties of quasi relative interior we refer to [33].

A convex cone $P \subset Y$ is called pointed if $P \cap(-P)=\{0\}$. Next lemma provides some properties of quasi relative interior that will be used in what follows.

LEMma 2.1. Let $\emptyset \neq M \subseteq Y$. The following relations hold:

(a) $0 \in \operatorname{qri}[\operatorname{co}(M \cup\{0\})] \Longleftrightarrow 0 \in \operatorname{qri}[\operatorname{cone}(\operatorname{co} M)]$;

(b) $0 \in \mathrm{qi}[\operatorname{co}(M \cup\{0\})] \quad \Longleftrightarrow 0 \in \mathrm{qi}[\operatorname{cone}(\operatorname{co} M)]$;

(c) $\operatorname{qri}(\operatorname{co} M)=\operatorname{co} M \cap \operatorname{qri}(\overline{\operatorname{co}} M)$;

(d) $\mathrm{qi}(\operatorname{co} M)=\operatorname{co} M \cap \mathrm{qi}(\overline{\mathrm{co}} M)$;

(e) If $\bar{M}$ is a convex set with $0 \in M$, then $0 \in \operatorname{qri}(\operatorname{co} M) \Longleftrightarrow 0 \in \operatorname{qri} \bar{M}$;

(f) If $\bar{M}$ is a convex set with $0 \in M$, then $0 \in \mathrm{qi}(\operatorname{co} M) \Longleftrightarrow 0 \in$ qi $\bar{M}$;

(g) If $M$ is a convex cone, then qri $M=M+$ qri $M$.

(h) If qri $M \neq \emptyset$, then $\overline{\text { qri } M}=\bar{M}$.

Proof. Statements $(a)$ and $(b)$ can be found in [16]. Statements $(c),(d),(g)$ and $(h)$ can be found in $[32,21,5,4]$.

Let us prove $(e)$. By assumptions, $\bar{M}=\overline{\mathrm{co}} M$, so that $(c)$ yields

$$
\operatorname{qri}(\operatorname{co} M)=\operatorname{co} M \cap \operatorname{qri} \bar{M}
$$

and since $0 \in M$, the thesis follows.

The proof of $(f)$ is analogous by exploiting $(d)$.

The positive polar of any set $A \subseteq Y$ is defined by:

$$
A^{*}:=\left\{y^{*} \in Y^{*}:\left\langle y^{*}, x\right\rangle \geq 0, \forall x \in A\right\}
$$

One immediately obtains that $A^{*}=(\bar{A})^{*}=(\text { co } A)^{*}=(\text { cone } A)^{*}$. Moreover, the bipolar theorem is obtained ([32, Theorem 1.1.9]):

$$
\overline{\mathrm{co}}(\text { cone } A)=\overline{\operatorname{cone}}(\operatorname{co} A)=A^{* *},
$$

where $A^{* *}:=\left(A^{*}\right)^{*}$.

We recall a simple generalization of a well-known result (see e.g., statement (v) at page 7 in [32], where it appears with slightly different assumptions).

Lemma 2.2. Let $A \subseteq Y$ and $B$ be a cone in $Y$. Then

$$
(A+B)^{*}=A^{*} \cap B^{*} .
$$


Proof. $(\subseteq)$. Let $x^{*} \in(A+B)^{*}$, then $\left\langle x^{*}, a+b\right\rangle \geq 0$ for all $a \in A, b \in b$,and since $B$ is a cone,

$$
\left\langle x^{*}, a+\lambda b\right\rangle \geq 0, \forall a \in A, \forall b \in B, \forall \lambda \geq 0
$$

Dividing by $\lambda>0$ in $(2.3)$ yields

$$
\left\langle x^{*}, \frac{1}{\lambda} a+b\right\rangle \geq 0, \forall a \in A, \forall b \in b, \forall \lambda>0
$$

and taking the limit for $\lambda \rightarrow+\infty$, we obtain $x^{*} \in B^{*}$. Setting $\lambda=0$ in (2.3) we have that $x^{*} \in A^{*}$.

$(\supseteq)$. It is straightforward.

The next result, which is new according to our best knowledge, characterizes quasi interior points of special sets, in particular for convex cones.

Proposition 2.3. Let $\emptyset \neq P$ be a convex cone. Then, $0 \in \mathrm{qi}(\operatorname{co} M+P)$ if, and only if

$$
0 \in \operatorname{co} M+P, \text { and } \forall p^{*} \in P^{*} \backslash\{0\}, \exists z \in M:\left\langle p^{*}, z\right\rangle<0 \text {. }
$$

As a consequence,

$$
p \in \text { qi } P \Longleftrightarrow p \in P \text { and }\left\langle p^{*}, p\right\rangle>0, \quad \forall p^{*} \in P^{*} \backslash\{0\} \text {. }
$$

In case $P$ is additionally closed,

$$
p \in \text { qi } P \Longleftrightarrow p \in Y \text { and }\left\langle p^{*}, p\right\rangle>0, \quad \forall p^{*} \in P^{*} \backslash\{0\} \text {. }
$$

Proof. Let us prove the "only if" part. Assume that there exists $p^{*} \in P^{*} \backslash\{0\}$ such that $\left\langle p^{*}, z\right\rangle \geq 0$ for all $z \in M$. The latter implies that $\left\langle p^{*}, z\right\rangle \geq 0$ for all $z \in$ co $M+P$. Thus, $\left\langle p^{*}, z\right\rangle \geq 0$ for all $z \in \overline{\operatorname{cone}}(\operatorname{co} M+P)=Y$, which gives $p^{*}=0$, yielding a contradiction. For the "if" part, we proceed as follows. Assume on the contrary that $N_{\text {co } M+P}(0) \neq\{0\}$. Then, there exists $p^{*} \in Y^{*}, p^{*} \neq 0$, satisfying $\left\langle p^{*}, z\right\rangle \leq 0$ for all $z \in$ co $M+P$. It follows that $-p^{*} \in P^{*}$ and $\left\langle-p^{*}, z\right\rangle \geq 0$ for all $z \in M$, which cannot happen.

When $P$ is additionally closed, the result follows by the fact that $P=P^{* *}$.

Next result can be found in [9].

Lemma 2.4. Let $M \subseteq Y$ be a nonempty set and $P \subseteq Y$ a convex cone with int $P \neq \emptyset$. Then

$$
M+\operatorname{int} P=\operatorname{int}(M+P)
$$

The following separation theorem was proved in [16] and it is an improvement of [5, Proposition 2.16] and [6, Theorem 2.7] since it does not rely on any convexity assumption.

Theorem 2.5. Let $\emptyset \neq M \subseteq Y$. Then, $0 \notin \operatorname{qri}[\operatorname{cone}(\operatorname{co} M)]$ (or, equivalently, $0 \notin$ $\operatorname{qri}[\operatorname{co}(M \cup\{0\})])$ if, and only if there exists $x^{*} \in Y^{*} \backslash\{0\}$ such that $\left\langle x^{*}, x\right\rangle \leq 0, \quad \forall x \in M$, with strict inequality for some $\tilde{x} \in M$. 
We recall a separation theorem for convex cones [3, Proposition 2] (see also [24, Theorem $3.22])$.

THEOREM 2.6. Let $A$ and $B$ two nonempty convex cones in the locally convex t.v.s. $Y$ such that $\bar{A} \cap \bar{B}=\{0\}$ and suppose that $B^{*}$ has nonempty interior in some topology $\tau$ such that $Y$ is the dual of $Y^{*}$. Then, $A^{*} \cap\left(-\operatorname{int} B^{*}\right) \neq \emptyset$.

Observe that the reverse implication also holds as stated in Theorem 3.22 of [24].

The following instance shows that one cannot substitute int $B^{*}$ by qi $B^{*}$ in the previous theorem without additional assumptions.

EXAMPLE 2.7. Let $\ell^{2}$ be the Hilbert space of all the real sequences $\left\{x_{n}\right\}$ with $\sum_{n=1}^{\infty} x_{n}^{2}<\infty$. Set

$A:=\left\{x=\left(x_{n}\right) \in \ell^{2}: x_{2 n+1}=x_{2 n}, n \in \mathbb{N}\right\}$ and $B:=\left\{x=\left(x_{n}\right) \in \ell_{+}^{2}: x_{2 n-1}=x_{2 n}, n \in \mathbb{N}\right\}$.

Then $A$ and $B$ are closed convex cones such that $\bar{A} \cap \bar{B}=\{0\}$ : indeed $x \in A \cap B$ implies $x_{n}$ is a non negative constant $k$ for every $n$, which does not belong to $\ell^{2}$ unless $k=0$. Moreover,

$A^{*}=\left\{x^{\prime} \in \ell^{2}: x_{1}^{\prime}=0, x_{2 n+1}^{\prime}+x_{2 n}^{\prime}=0, n \in \mathbb{N}\right\}, \quad B^{*}=\left\{x^{\prime} \in \ell^{2}: x_{2 n-1}^{\prime}+x_{2 n}^{\prime} \geq 0, n \in \mathbb{N}\right\}$,

qi $B^{*}=\left\{x^{\prime} \in \ell^{2}: x_{2 n-1}^{\prime}+x_{2 n}^{\prime}>0, n \in \mathbb{N}\right\}$.

In fact, $\left\langle x^{\prime}, x\right\rangle \geq 0$, for all $x \in A$, leads to the condition

$$
x_{2 n+1}^{\prime} x_{2 n+1}+x_{2 n}^{\prime} x_{2 n}=\left(x_{2 n+1}^{\prime}+x_{2 n}^{\prime}\right) x_{2 n} \geq 0, \quad n \in \mathbb{N},
$$

which implies $x_{2 n+1}^{\prime}+x_{2 n}^{\prime}=0$ for all $n \in \mathbb{N}$. Note that since the first element of the sequence $\left(x_{n}\right) \in A$ is arbitrary, then $x_{1}^{\prime}=0$.

$A$ similar reasoning holds for $B^{*}$, noticing that $B \subseteq \ell_{+}^{2}$. Next, we prove that $A^{*} \cap\left(-\right.$ qi $\left.B^{*}\right)=\emptyset$. Suppose that $x^{\prime} \in A^{*} \cap\left(-\mathrm{qi} B^{*}\right)$, then

$$
x_{1}^{\prime}=0, \quad x_{2 n+1}^{\prime}+x_{2 n}^{\prime}=0, \quad x_{2 n-1}^{\prime}+x_{2 n}^{\prime}<0, n \in \mathbb{N} .
$$

It follows that $x_{2 n+1}^{\prime}>x_{2 n-1}^{\prime}$, i.e., $x_{2 n+1}^{\prime}$ is a positive increasing sequence and $x_{2 n}^{\prime}$ is negative decreasing. Therefore $\left(x_{n}^{\prime}\right)^{2} \nrightarrow 0$, and so $x^{\prime} \notin \ell^{2}$, a contradiction.

In spite of the previous example, we report a further result due to Borwein which involves quasi-interior of $B$ provided this set is locally compact.

Proposition 2.8. [3, Proposition 3] Suppose A, B are closed convex cones in $Y$ such that $A \cap B=\{0\}$. Suppose that $B$ is pointed and locally compact (i.e., has a compact neighborhood base in the relative topology on $B)$. Then $A^{*} \cap\left(-\right.$ qi $\left.B^{*}\right) \neq \emptyset$.

We note that the set $B$ defined in Example 2.7 is a closed convex pointed cone but it is not locally compact, so that Proposition 2.8 cannot be applied. Indeed, since $\ell^{2}$ is a Hilbert space and $\operatorname{int}\left(B^{*}\right)=\emptyset$, then the set $B$ cannot have a bounded base (see [23] Theorem 3.8.4 ) as implied by the local compactness of $B$ (see, (2.4) [25]). For completeness, we recall that, in case $Y$ is a normed space, Theorem 3.8.4 [23] states that $B$ has a bounded base iff $B^{*}$ has an interior point with respect to the norm topology for $Y^{*}$ which in the present case can be identified with $Y$. 
3. The vector optimization problem and saddle points . In this section we first characterize the optimality conditions for the vector problem (1.1), by introducing suitable subsets of the image space associated with (1.1), namely the space $Y \times Z$ where the objective and the constraints functions run. Separation techniques in the image space allow us to derive saddle point conditions for the generalized Lagrangian function associated with (1.1) and defined by (3.7).

As mentioned in the introduction, problem (1.1) allows us to deal in a unified manner with several optimality concepts. The set of vectors $\bar{x} \in K$ satisfying (1.1) is denoted by $E_{S}=E_{S}(K)$, and each one of its elements is called a (global) $S$-minimizer of $f$ on $K$. It is obvious that if $0 \notin S$, then (1.1) reduces to

$$
\bar{x} \in K, \quad f(x)-f(\bar{x}) \notin-S, \quad \forall x \in K .
$$

In case $0 \in S$, then

$$
\bar{x} \in E_{S} \Longrightarrow f(x) \neq f(\bar{x}) \forall x \in K, x \neq \bar{x}
$$

In practice, a convex cone $P$ is prescribed, and $S \subseteq Y$ fulfils the following condition

$$
S+P \subseteq S
$$

and this is equivalent to $S+P=S$. By specializing the set $S$ we recover most of the classic definitions of efficiency for a vector optimization problem. When an ordering convex cone $P$ such that $\bar{P} \neq Y$ is present, several preference relations may be introduced. For instance:

- $S:=Y \backslash-P$ leads to ideal or strong solutions for (1.1), and in such a case, we denote $E_{S}$ simply by $E_{i}$;

- $S:=P \backslash(-P)=P \backslash(P \cap-P)$ gives rise to efficient solutions for (1.1), and in such a case, we denote $E_{S}$ shortly by $E$;

- $S:=P$ yields strictly efficient solutions for (1.1), and in such a case, we denote $E_{S}$ simply by $E_{1}$;

- $S:=$ qri $P$, with $0 \notin$ qri $P$, leads to weakly efficient solutions for (1.1), and in this case $E_{S}$ is denoted by $E_{W}$.

One can check that, for such a convex cone $P$,

$$
\left.E_{i} \subseteq E \subseteq E_{W} \text { (here qri } P \neq \emptyset\right)
$$

We will check later on that all the preceding specializations of $S$ satisfy (3.1). On the other hand, observe that for the first two and the fourth instances $0 \notin S$; whereas for the third one, $0 \in S$. For a more detailed discussion on the possible choices of the set $S$, we refer to $[14,15]$. We must point out that we will deal with (Benson) proper efficient solutions even if such a notion cannot be expressed in terms of any $S$.

It is not difficult to show that condition (3.1) implies those cited in the following lemma.

Lemma 3.1. Let $P$ be a (not necessarily convex) cone in $Y$. Let us consider the following assertions:

(a) $S+P=S$; 

(b) $\bar{S}=\bar{S}+\bar{P}$;
(c) $S^{*}=(\bar{S}+\bar{P})^{*}$;
(d) $S^{*} \subseteq P^{*}$.

Then,

$$
(a) \Longrightarrow(b) \Longrightarrow(c) \Longleftrightarrow(d)
$$

Proof. $(a) \Rightarrow(b)$ : By assumption, we get

$$
\bar{S} \subseteq \bar{S}+\bar{P} \subseteq \overline{S+P}=\bar{S}
$$

Thus $\bar{S}=\bar{S}+\bar{P}$.

$(b) \Rightarrow(c)$ : By $(b),(\bar{S})^{*}=(\bar{S}+\bar{P})^{*}$, and since $S^{*}=(\bar{S})^{*}$, the result follows.

$(c) \Rightarrow(d)$ : By $(c)$ and $(2.2)$ one obtains

$$
S^{*}=(\bar{S}+\bar{P})^{*}=\bar{S}^{*} \cap \bar{P}^{*}=S^{*} \cap P^{*},
$$

which proves our assertion.

$(d) \Rightarrow(c):(d)$ and $(2.2)$ imply that $S^{*}=S^{*} \cap P^{*}=(S+P)^{*}=(\overline{S+P})^{*}$. Since $S+P \subseteq$ $\bar{S}+\bar{P} \subseteq \overline{S+P}$, then the following inclusions hold:

$$
S^{*}=(S+P)^{*} \supseteq(\bar{S}+\bar{P})^{*} \supseteq(\overline{S+P})^{*}=S^{*},
$$

which completes the proof. $\square$

REMARK 3.2. The opposite implications in Lemma 3.1 do not hold as the following examples show.

Set $P:=\mathbb{R}_{+} \times\{0\}, S:=\left(\right.$ int $\left.\mathbb{R}_{+}^{2}\right) \cup\{(0,0)\}$, then (b) is fulfilled but (a) does not hold. For the other implication, set $P:=\left\{\left(x_{1}, x_{2}\right) \in \mathbb{R}_{+}^{2}: x_{1}=x_{2}\right\}$ and

$$
S:=\left\{\left(x_{1}, x_{2}\right) \in \mathbb{R}_{+}^{2}: x_{1} x_{2}=0, x_{1} \geq 1, x_{2} \geq 1\right\} \cup\left\{\left(x_{1}, x_{2}\right) \in \mathbb{R}_{+}^{2}: x_{1}+x_{2}=1\right\} .
$$

Note that $S+P=\bar{S}+\bar{P}=\overline{\mathrm{co}} S \neq \bar{S}=S$, therefore (b) and (a) do not hold, but (c) is fulfilled, since $S^{*}=(S+P)^{*}=(\bar{S}+\bar{P})^{*}$ and cone $(S+P)=$ cone $S=\mathbb{R}_{+}^{2} \supseteq P$.

Let $\bar{x} \in K, F:=(f, g)$ and consider the sets:

$$
\begin{gathered}
\hat{\mathcal{E}}_{\bar{x}}:=F(C \backslash\{\bar{x}\})-(f(\bar{x}), 0)+(P \times Q) . \\
\mathcal{E}_{\bar{x}}:=F(C)-(f(\bar{x}), 0)+(P \times Q) .
\end{gathered}
$$

It is easy to check that

$$
\begin{aligned}
(F(C \backslash\{\bar{x}\})-(f(\bar{x}), 0)) \cap-(S \times Q)=\emptyset & \Longleftrightarrow \hat{\mathcal{E}}_{\bar{x}} \cap-(S \times Q)=\emptyset \\
& \Longleftrightarrow \hat{\mathcal{E}}_{\bar{x}} \cap-(S \times\{0\})=\emptyset .
\end{aligned}
$$

Similarly, we have

$$
\begin{aligned}
(F(C)-(f(\bar{x}), 0)) \cap-(S \times Q)=\emptyset & \Longleftrightarrow \mathcal{E}_{\bar{x}} \cap-(S \times Q)=\emptyset \\
& \Longleftrightarrow \mathcal{E}_{\bar{x}} \cap-(S \times\{0\})=\emptyset .
\end{aligned}
$$


Proposition 3.3. Assume that (3.1) holds. Then, $\bar{x} \in E_{S}$ if, and only if

$$
\hat{\mathcal{E}}_{\bar{x}} \cap-(S \times\{0\})=\emptyset .
$$

Furthermore, if $0 \notin S$, then (3.4) is equivalent to

$$
\mathcal{E}_{\bar{x}} \cap-(S \times\{0\})=\emptyset .
$$

Proof. We observe that $\bar{x} \in E_{S}$ iff the following system is impossible:

$$
f(x)-f(\bar{x}) \in-S, \quad g(x) \in-Q, x \in C, x \neq \bar{x},
$$

which can be written as,

$$
(F(C \backslash\{\bar{x}\})-(f(\bar{x}), 0)) \cap-(S \times Q)=\emptyset .
$$

Since $(F(C \backslash\{\bar{x}\})-(f(\bar{x}), 0)) \subseteq \hat{\mathcal{E}}_{\bar{x}}$ then obviously (3.4) implies (3.6). For the reverse implication, assume that $(3.4)$ does not hold, i.e., $0 \in \hat{\mathcal{E}}_{\bar{x}}+(S \times Q)$. Then, since $Q$ is a convex cone and by the assumptions on the set $S$, the following relations hold:

$$
0 \in(F(C \backslash\{\bar{x}\})-(f(\bar{x}), 0))+(P \times Q)+(S \times Q)=(F(C \backslash\{\bar{x}\})-(f(\bar{x}), 0))+(S \times Q)
$$

Therefore, $0 \in F(C \backslash\{\bar{x}\})-(f(\bar{x}), 0))+(S \times Q)$, which contradicts (3.6) and the proof is complete.

Let $Y^{*}$ be the topological dual space of $Y$ and denote by $\langle\cdot, \cdot\rangle$ the duality pairing between $Y$ and $Y^{*}$ and similarly for $Z$ and $Z^{*}$. The generalized Lagrangian function $L: Y^{*} \times Z^{*} \times C \rightarrow \mathbb{R}$ associated with (1.1) is defined by

$$
L(\theta, \lambda, x) \doteq\langle\theta, f(x)\rangle+\langle\lambda, g(x)\rangle
$$

Given $\theta^{*} \in Y^{*}$, we say that $\left(\lambda^{*}, \bar{x}\right) \in Q^{*} \times C$ is a saddle point for $L\left(\theta^{*}, \lambda, x\right)$ on $Q^{*} \times C$, if,

$$
L\left(\theta^{*}, \lambda, \bar{x}\right) \leq L\left(\theta^{*}, \lambda^{*}, \bar{x}\right) \leq L\left(\theta^{*}, \lambda^{*}, x\right), \quad \forall(\lambda, x) \in Q^{*} \times C .
$$

Next proposition, which is well known in the context of scalar constrained optimization, provides a useful characterization of the saddle point condition (see e.g. [26], Proposition 5.2).

Proposition 3.4. Let $Z$ be a locally convex t.v.s., and let $\theta^{*} \in Y^{*}$. Then $\left(\lambda^{*}, \bar{x}\right) \in Q^{*} \times C$ is a saddle point for $L\left(\theta^{*}, \lambda, x\right)$ on $Q^{*} \times C$, if, and only if

(a) $L\left(\theta^{*}, \lambda^{*}, \bar{x}\right) \leq L\left(\theta^{*}, \lambda^{*}, x\right), \quad \forall x \in C$;

(b) $g(\bar{x}) \in-Q$;

(c) $\left\langle\lambda^{*}, g(\bar{x})\right\rangle=0$.

3.1. The main results. Next theorem states necessary and/or sufficient conditions for the existence of a saddle point with the requirement that the multiplier $\theta^{*}$ belongs to qi $S^{*}$, and so, a fortiori (Theorem 4.1), conditions for $\bar{x} \in K$ to be in $E_{S}$, which will be established in Section 4.

Notice that

qi $S^{*} \neq \emptyset \Longrightarrow \overline{\text { cone }}($ co $S)$ is pointed. 
Conversely, by the Krein-Rutman theorem (see, e.g., [24, Theorem 3.38]), if $Y$ is a separable Banach space and $K \subset Y$ is a closed pointed convex cone, then qi $K^{*} \neq \emptyset$.

Theorem 3.5. Assume that $Y$ and $Z$ are locally convex t.v.s., $P \subseteq Y$ is a convex cone, and $S \subseteq Y$ is a nonempty set satisfying $S+P \subseteq S$. Let us consider the following assertions:

(a) There exist $\theta^{*} \in$ qi $S^{*}$ and $\lambda^{*} \in Q^{*}$ such that $\left(\lambda^{*}, \bar{x}\right)$ is a saddle point for $L\left(\theta^{*}, \lambda, x\right)$ on $Q^{*} \times C$;

(b) $\bar{x} \in K$, $\overline{\operatorname{cone}}(\operatorname{co} S)$ is pointed and

$$
\overline{\text { cone }}\left(\operatorname{co~} \mathcal{E}_{\bar{x}}\right) \cap-(\overline{\operatorname{cone}}(\cos S) \backslash\{0\}) \times\{0\}=\emptyset ;
$$

Then, $(a) \Longrightarrow(b)$. Additionally, $(b) \Longrightarrow(a)$ under any of the following circumstances:

(c) A topology is given on $Y \times Z$ such that $Y \times Z$ is the dual of $(Y \times Z)^{*}$ and moreover, $\theta^{*} \in \operatorname{int} S^{*}$ in $(a)$, provided that $S^{*}$ has nonempty interior;

(d) $\overline{\text { cone }}($ co $S)$ is locally compact.

Proof. $(a) \Rightarrow(b)$ : By (3.9), $\overline{\operatorname{cone}}(\operatorname{co} S)$ is pointed. By Lemma 3.1, $S^{*} \subseteq P^{*}$ and by Proposition $3.4(\mathrm{~b}), \bar{x} \in K$. The second inequality in (3.8) yields:

$$
\left\langle\theta^{*}, f(x)-f(\bar{x})\right\rangle+\left\langle\lambda^{*}, g(x)-g(\bar{x})\right\rangle \geq 0, \quad \forall x \in C,
$$

which is equivalent to

$$
\left\langle\theta^{*}, f(x)-f(\bar{x})+p\right\rangle+\left\langle\lambda^{*}, g(x)+q\right\rangle \geq 0, \quad \forall x \in C, \forall p \in P, \forall q \in Q
$$

because of $\theta^{*} \in S^{*} \subseteq P^{*}, \lambda^{*} \in Q^{*}$ and Proposition 3.4 (c). Moreover, observe that (3.11) is equivalent to

$$
\left\langle\theta^{*}, u\right\rangle+\left\langle\lambda^{*}, v\right\rangle \geq 0, \quad \forall(u, v) \in \overline{\operatorname{cone}}\left(\operatorname{co} \mathcal{E}_{\bar{x}}\right) .
$$

Since $\theta^{*} \in$ qi $S^{*}$, Proposition 2.3 implies

$$
\left\langle\theta^{*}, u\right\rangle>0, \quad \forall u \in S^{* *} \backslash
$$

and, therefore,

$$
\left\langle\theta^{*}, u\right\rangle+\left\langle\lambda^{*}, v\right\rangle<0, \quad \forall(u, v) \in-(\overline{\operatorname{cone}}(\cos S) \backslash\{0\}) \times\{0\},
$$

so that $(b)$ follows.

$(b) \Rightarrow(a)$ under $(c)$ : We preliminarly notice that, by Lemma 3.21 (d) in [24] and taking into account Proposition 2.3, then $(c)$ yields int $S^{*}=$ qi $S^{*}$.

As (b) holds, then

$$
\overline{\operatorname{cone}}\left(\operatorname{co} \mathcal{E}_{\bar{x}}\right) \cap-(\overline{\operatorname{cone}}(\cos S) \times\{0\})=\{(0,0)\} .
$$

Applying Theorem 2.6 with $A \doteq \operatorname{cone}\left(\operatorname{co} \mathcal{E}_{\bar{x}}\right)$ and $B \doteq-\overline{\operatorname{cone}}(\operatorname{co} S) \times\{0\}$, it follows that there exists $\left(\theta^{*}, \lambda^{*}\right) \in \operatorname{int}(\overline{\operatorname{cone}}(\cos S) \times\{0\})^{*}=\operatorname{int}\left[(\overline{\operatorname{cone}}(\cos S))^{*} \times Z^{*}\right]=\operatorname{int}\left(S^{*} \times Z^{*}\right)=\left(\operatorname{int} S^{*}\right) \times Z^{*}$, such that

$$
\left\langle\theta^{*}, u\right\rangle+\left\langle\lambda^{*}, v\right\rangle \geq 0, \quad \forall(u, v) \in \operatorname{cone}\left(\operatorname{co} \mathcal{E}_{\bar{x}}\right)
$$


As shown in the previous implication, (3.12) is equivalent to (3.11). Computing (3.11) for $x=\bar{x}$ and $p=0$ we get

$$
\left\langle\lambda^{*}, g(\bar{x})\right\rangle+\left\langle\lambda^{*}, q\right\rangle \geq 0, \quad \forall q \in Q
$$

which implies $\lambda^{*} \in Q^{*}$ and consequently, since $g(\bar{x}) \in-Q$, it follows that $\left\langle\lambda^{*}, g(\bar{x})\right\rangle=0$. Finally, (3.11) computed for $p=0$ and $q=0$ leads to (3.10) and applying Proposition 3.4 we complete the proof.

$(b) \Rightarrow(a)$ under $(d)$ : It is similar to the previous one. Thus,

$$
\overline{\text { cone }}\left(\operatorname{co} \mathcal{E}_{\bar{x}}\right) \cap-(\overline{\operatorname{cone}}(\cos S) \times\{0\})=\{(0,0)\} .
$$

We now apply Proposition 2.8 with $A \doteq \overline{\operatorname{cone}}\left(\operatorname{co} \mathcal{E}_{\bar{x}}\right)$ and $B \doteq-\overline{\operatorname{cone}}(\cos S) \times\{0\}$. By recalling that $\operatorname{qri}(A \times B)=\operatorname{qri}(A) \times \operatorname{qri}(B)$ (see [5]), it follows that there exists $\left(\theta^{*}, \lambda^{*}\right) \in \operatorname{qi}(\bar{P} \times\{0\})^{*}=$ $\mathrm{qi}\left(P^{*} \times Z^{*}\right)=\left(\right.$ qi $\left.P^{*}\right) \times Z^{*}$, such that $(3.12)$ holds. The rest of the proof is the same as above.

When $Y=\mathbb{R}, S=\mathbb{R}_{+} \backslash\{0\}$, then the equivalence between (a) and (b) stated by Theorem 3.5 collapses to Theorem 3.2 of [16] under the further assumption that the given constrained extremum problem admits an optimal solution.

We consider the particular case of Theorem 3.5 where $g=0, Q=0$. Hence $K=C \subseteq X$ and we set $\mathcal{E}_{0 \bar{x}}:=f(C)-f(\bar{x})+P$.

Then, assuming int $S^{*} \neq \emptyset$ in some topology such that $Y$ is the dual of $Y^{*}$, Theorem 3.5 reads as follows:

Theorem 3.6. Assume that $S \subseteq Y$ is a nonempty set and int $S^{*} \neq \emptyset$ in some topology such that $Y$ is the dual of $Y^{*}, S+P \subseteq S$ with $P$ being a convex cone.

The following assertions are equivalent:

(a) $\bar{x} \in \bigcup_{\theta^{*} \in \operatorname{int} S^{*}}^{\operatorname{argmin}}\left\langle\theta^{*}, f(\cdot)\right\rangle$;

(b) $\bar{x} \in C$, $\overline{\text { cone }}(\cos S)$ is pointed and $\overline{\operatorname{cone}}\left(\cos \mathcal{E}_{0 \bar{x}}\right) \cap-(\overline{\operatorname{cone}}(\cos S))=\{0\}$.

We can still go further. Let us consider $S=P$ with $P$ being a closed convex cone. Then, the equality in $(b)$ of Theorem 3.6 becomes

$$
\overline{\operatorname{cone}}(\cos f(C)-f(\bar{x})+P) \cap-P=\{0\},
$$

which, by deleting the convex hull, is exactly the definition for $\bar{x}$ to be a (Benson) properly efficient solution to problem (1.1) without explicit constraints.

We recall that $\bar{x} \in C$ is a (Benson) properly efficient point of $f$ on $C([1])$, in short $\bar{x} \in E_{p r}$, if

$$
\overline{\mathrm{cone}}(f(C)-f(\bar{x})+P) \cap(-P)=\{0\} .
$$

One can easily check that if $E_{p r}$ is nonempty, then $P$ is pointed.

Motivated by the previous remark, we can introduce a notion of (generalized) proper efficiency for a vector problem with explicit constraints. This notion arises from the equality in $(b)$ of Theorem 3.5 after deleting the convex hull, namely: 
Definition 3.7. We say $\bar{x} \in K$ is a proper efficient solution to problem (1.1), if $\overline{\operatorname{cone}}(\operatorname{co} S)$ is pointed and

$$
\overline{\operatorname{cone}}\left(\mathcal{E}_{\bar{x}}\right) \cap-(\overline{\operatorname{cone}}(\cos S) \backslash\{0\}) \times\{0\}=\emptyset .
$$

A stronger notion of proper efficiency may be given if we substitute $\mathcal{E}_{\bar{x}}$ by co $\mathcal{E}_{\bar{x}}$.

We come back to discuss proper efficiency later on.

3.2. Characterizing saddle points via generalized interiority notions. We start by establishing a general necessary and sufficient condition for the existence of a saddle point for $L(\theta, \cdot, \cdot)$ on $Q^{*} \times C$ via quasi interior notions.

TheOREM 3.8. Assume that $Y$ and $Z$ are locally convex t.v.s., $P \subseteq Y$ be a convex cone, $S \subseteq Y$ is a nonempty set satisfying $S+P \subseteq S$, and $\overline{\mathrm{cone}}(\operatorname{co} S)=\bar{P}$. The following assertions are equivalent:

(a) There exist $\theta^{*} \in S^{*}$ and $\lambda^{*} \in Q^{*},\left(\theta^{*}, \lambda^{*}\right) \neq(0,0)$, such that $\left(\lambda^{*}, \bar{x}\right)$ is a saddle point for $L\left(\theta^{*}, \lambda, x\right)$ on $Q^{*} \times C$;

(b) $\bar{x} \in K$ and $(0,0) \notin \mathrm{qi}\left[\operatorname{cone}\left(\operatorname{co} \mathcal{E}_{\bar{x}}\right)\right]$;

(c) $\bar{x} \in K$ and $(0,0) \notin \mathrm{qi}\left[\operatorname{co}\left(\mathcal{E}_{\bar{x}}\right)\right]$.

Proof. We preliminarly note that if $\bar{x} \in K$, then $(0,0) \in \mathcal{E}_{\bar{x}}$. Moreover, by (b) of Lemma 2.1, it follows that $(b)$ and $(c)$ are equivalent. Assume that $(a)$ is fulfilled. By Proposition 3.4 (b), it follows that $\bar{x} \in K$. As in the proof of Theorem 3.5 the saddle point condition leads to the inequality:

$$
-\left\langle\theta^{*}, u\right\rangle-\left\langle\lambda^{*}, v\right\rangle \leq 0, \forall(u, v) \in \operatorname{co} \mathcal{E}_{\bar{x}}
$$

i.e., $(0,0) \neq\left(-\theta^{*},-\lambda^{*}\right) \in N_{\text {co } \mathcal{E}_{\bar{x}}}(0,0)$ and, since $(0,0) \in\left(\operatorname{co} \mathcal{E}_{\bar{x}}\right)$, then $(c)$ holds.

Conversely, if $(c)$ is fulfilled, then there exists $(0,0) \neq\left(-\theta^{*},-\lambda^{*}\right) \in N_{\text {co }} \mathcal{E}_{\bar{x}}(0,0)$ and (3.14) holds. As in Theorem 3.5 (3.14) is equivalent to (3.11), i.e.,

$$
\left\langle\theta^{*}, f(x)-f(\bar{x})+p\right\rangle+\left\langle\lambda^{*}, g(x)+q\right\rangle \geq 0, \quad \forall x \in C, \forall p \in P, \forall q \in Q,
$$

which implies that $\theta \in P^{*}=S^{*}$ and $\lambda^{*} \in Q^{*}$. Note that the assumption $\overline{\operatorname{cone}}(\operatorname{co} S)=\bar{P}$, implies that (is equivalent to) $P^{*}=S^{*}$.

Setting $x \doteq \bar{x}, p=0$ in (3.11), with the same arguments used in Theorem 3.5, we can prove that $\lambda^{*} \in Q^{*}$ and $\left\langle\lambda^{*}, g(\bar{x})\right\rangle=0$, which yields (3.10). Now, all the assumptions (a)-(c) of Proposition 3.4 are fulfilled and $\left(\lambda^{*}, \bar{x}\right)$ is a saddle point for $L\left(\theta^{*}, \lambda, x\right)$ on $Q^{*} \times C$.

In the final part of this section, we will show that the multiplier $\theta^{*}$, associated with the objective function in (3.7), does not vanish under the following Slater-type constraints qualifications introduced in [16]:

$$
\begin{aligned}
& 0 \in \operatorname{qri}(\operatorname{co}(g(C)+Q)) \\
& 0 \in \operatorname{qi}(\operatorname{co}(g(C)+Q))
\end{aligned}
$$


Obviously (3.16) implies (3.15).

As it will be recalled in the next section, it is known that the existence of a saddle point for the Lagrangian function, under the assumption that the multiplier $\theta^{*} \in P^{*} \backslash\{0\}$, is a sufficient optimality condition for a weak efficient solution of (1.1).

TheOREM 3.9. Assume that $Y$ and $Z$ are locally convex t.v.s., $P \subseteq Y$ be a convex cone, $S \subseteq Y$ is a nonempty set satisfying $S+P \subseteq S, \overline{\operatorname{cone}}(\operatorname{co} S)=\bar{P}$ and (3.15) is fulfilled. Then, the following assertions are equivalent:

(a) There exist $\theta^{*} \in S^{*} \backslash\{0\}, \tilde{x} \in C, \tilde{p} \in P, \tilde{q} \in Q$ and $\lambda^{*} \in Q^{*}$ such that $\left(\lambda^{*}, \bar{x}\right)$ is a saddle point for $L\left(\theta^{*}, \lambda, x\right)$ on $Q^{*} \times C$ and, moreover,

$$
\left\langle\theta^{*}, f(\tilde{x})-f(\bar{x})+\tilde{p}\right\rangle+\left\langle\lambda^{*}, g(\tilde{x})+\tilde{q}\right\rangle>0
$$

(b) $\bar{x} \in K$ and $(0,0) \notin \operatorname{qri}\left[\operatorname{cone}\left(\cos \mathcal{E}_{\bar{x}}\right)\right]$;

(c) $\bar{x} \in K$ and $(0,0) \notin \operatorname{qri}\left[\operatorname{co} \mathcal{E}_{\bar{x}}\right]$.

Proof. By (a) of Lemma 2.1, it follows that (b) and (c) are equivalent, taking into account that $\bar{x} \in K$ implies that $(0,0) \in \mathcal{E}_{\bar{x}}$. Assume that $(a)$ is fulfilled. By Proposition 3.4 (b) it follows that $\bar{x} \in K$. As already observed, the saddle point condition implies that $\bar{x} \in K$ and the inequalities (3.11) and

$$
-\left\langle\theta^{*}, u\right\rangle-\left\langle\lambda^{*}, v\right\rangle \leq 0, \forall(u, v) \in \mathcal{E}_{\bar{x}},
$$

By assumption, we have that strict inequality holds in (3.17) for $u=f(\tilde{x})-f(\bar{x})+\tilde{p}, v=g(\tilde{x})+\tilde{q}$, and by Theorem 2.5, where we set $M \doteq \mathcal{E}_{\bar{x}}$, we obtain that (b) is fulfilled.

Conversely, assume that (b) holds. Theorem 2.5 yields that there exists $\left(\theta^{*}, \lambda^{*}\right) \in(Y \times Z)^{*}$ such that

$$
-\left\langle\theta^{*}, u\right\rangle-\left\langle\lambda^{*}, v\right\rangle \leq 0, \forall(u, v) \in \mathcal{E}_{\bar{x}},
$$

with strict inequality for some $(\tilde{u}, \tilde{v}) \in \mathcal{E}_{\bar{x}}$, i.e,

$$
\left\langle\theta^{*}, f(x)-f(\bar{x})+p\right\rangle+\left\langle\lambda^{*}, g(x)+q\right\rangle \geq 0, \quad \forall p \in P, \forall x \in C, \forall q \in Q,
$$

with strict inequality for some $\tilde{x} \in C, \tilde{p} \in P, \tilde{q} \in Q$.

Arguing as in the proof of Theorem 3.5 we can show that $\left(\lambda^{*}, \bar{x}\right)$ is a saddle point for $L\left(\theta^{*}, \lambda, x\right)$ on $Q^{*} \times C$ : we have only to show that $\theta^{*} \neq 0$ in $(3.18)$.

By contradiction, suppose that $\theta^{*}=0$ in (3.18); then $\lambda^{*} \neq 0$ and (3.18) becomes:

$$
\left\langle\lambda^{*}, g(x)+q\right\rangle \geq 0, \forall x \in C, \forall q \in Q
$$

or, equivalently, $\quad\left\langle\lambda^{*}, v\right\rangle \geq 0, \quad \forall v \in g(C)+Q$,

$$
\left\langle\lambda^{*}, v\right\rangle \geq 0, \quad \forall v \in \operatorname{co}(g(C)+Q),
$$

i.e. $-\lambda^{*} \in N_{\operatorname{co}(g(C)+Q)}(0)$ (note that, since $\bar{x} \in K$, then $0 \in \operatorname{co}(g(C)+Q)$ ). Assumption (3.15) is equivalent to say that $N_{\operatorname{co}(g(C)+Q)}(0)$ is a linear subspace in $Z$, which implies that $\lambda^{*} \in N_{\operatorname{co}(g(C)+Q)}(0)$. This contradicts the strict inequality

$$
\left\langle\lambda^{*}, \bar{v}\right\rangle>0, \quad \bar{v} \doteq g(\tilde{x})+\tilde{q},
$$


and the proof is complete.

Theorem 3.10. Assume that $Y$ and $Z$ are locally convex t.v.s., $P \subseteq Y$ be a convex cone, $S \subseteq Y$ is a nonempty set satisfying $S+P \subseteq S, \overline{\operatorname{cone}}(\operatorname{co} S)=\bar{P}$ and assume that (3.16) is fulfilled. Then, the following assertions are equivalent:

(a) There exist $\theta^{*} \in S^{*} \backslash\{0\}$ and $\lambda^{*} \in Q^{*}$ such that $\left(\lambda^{*}, \bar{x}\right)$ is a saddle point for $L\left(\theta^{*} ; \lambda, x\right)$ on $Q^{*} \times C$;

(b) $\bar{x} \in K$ and $(0,0) \notin \mathrm{qi}\left[\operatorname{cone}\left(\operatorname{co} \mathcal{E}_{\bar{x}}\right)\right]$;

(c) $\bar{x} \in K$ and $(0,0) \notin \mathrm{qi}\left[\operatorname{co}\left(\mathcal{E}_{\bar{x}}\right)\right]$.

Proof. By (b) of Lemma 2.1, it follows that $(b)$ and $(c)$ are equivalent, noticing that since $\bar{x} \in K$, then $(0,0) \in \mathcal{E}_{\bar{x}}$. Assume that $(a)$ is fulfilled. Since (3.16) implies (3.15), by Theorem 3.9 it follows that $(0,0) \notin \operatorname{qri}\left[\operatorname{cone}\left(\operatorname{co} \mathcal{E}_{\bar{x}}\right)\right]$ and $\bar{x} \in K$, which implies (b).

Conversely, assume that (b) holds. Theorem 3.8 guarantees that there exist $\theta^{*} \in S^{*}$ and $\lambda^{*} \in Q^{*},\left(\theta^{*}, \lambda^{*}\right) \neq(0,0)$, such that $\left(\lambda^{*}, \bar{x}\right)$ is a saddle point for $L\left(\theta^{*}, \lambda, x\right)$ on $Q^{*} \times C$. We have only to show that $\theta^{*} \neq 0$. As observed in the proof of Theorem 3.5, the saddle point condition leads to the inequality (3.11). By contradiction, suppose that $\theta^{*}=0$ in (3.11). Then $\lambda^{*} \neq 0$ and (3.11) becomes:

$$
\left\langle\lambda^{*}, g(x)+q\right\rangle \geq 0, \forall x \in C, \forall q \in Q
$$

i.e.,

$$
\left\langle\lambda^{*}, v\right\rangle \geq 0, \quad \forall v \in g(C)+Q
$$

or, equivalently,

$$
\left\langle\lambda^{*}, v\right\rangle \geq 0, \quad \forall v \in \overline{\operatorname{cone}}(\operatorname{co}(g(C)+Q))
$$

By assumption (3.16), $\overline{\operatorname{cone}}(\operatorname{co}(g(C)+Q))=Z$, which contradicts the previous inequality and the proof is complete.

Theorems 3.9 and 3.10 extend to the vector case Theorems 3.11 and 3.10 of [16], respectively.

4. Necessary or sufficient optimality conditions for efficiency in terms of saddle points. It is well known that saddle point conditions are a source of sufficient optimality conditions for (1.1), provided that the multiplier $\theta^{*}$ associated with the objective function fulfils suitable properties. In this direction, such conditions for vector problems involving cones having nonempty topological interior were given in $[2,29]$.

Next result encompasses both notions of efficiency and weak efficiency, where the latter is defined in terms of quasi-interior. Throughout this section we will assume that $P$ is a convex cone.

Theorem 4.1. Assume that (3.1) is fulfilled. If any of the following conditions hold:

(a) $S \subseteq$ qi $P \neq \emptyset$ and there exist $\theta^{*} \in P^{*} \backslash\{0\}$ and $\lambda^{*} \in Q^{*}$ such that $\left(\lambda^{*}, \bar{x}\right)$ is a saddle point for $L\left(\theta^{*}, \lambda, x\right)$ on $Q^{*} \times C$;

(b) $\overline{\operatorname{cone}}(\operatorname{co} S)=\bar{P}, 0 \notin S$ and there exist $\theta^{*} \in$ qi $P^{*}$ and $\lambda^{*} \in Q^{*}$ such that $\left(\lambda^{*}, \bar{x}\right)$ is a saddle point for $L\left(\theta^{*}, \lambda, x\right)$ on $Q^{*} \times C$; 
then $\bar{x} \in E_{S}$.

Proof. Assume that (a) holds. We preliminarly note that $0 \notin S$, otherwise we would have $0 \in$ qi $P$, i.e., $\bar{P}=Y$ and therefore $P^{*}=\{0\}$, which contradicts the assumptions.

Proposition 3.3 yields that $\bar{x} \in E_{S}$ if and only if (3.4) is fulfilled. As in the proof of Theorem 3.5 , if $\left(\lambda^{*}, \bar{x}\right)$ is a saddle point for $L\left(\theta^{*}, \lambda, x\right)$ on $Q^{*} \times C$, then

$$
\left\langle\theta^{*}, u\right\rangle+\left\langle\lambda^{*}, v\right\rangle \geq 0, \quad \forall(u, v) \in \mathcal{E}_{\bar{x}} .
$$

Since $\theta^{*} \in P^{*} \backslash\{0\}$ and $\lambda^{*} \in Q^{*}$, then (2.4) yields:

$$
\left\langle\theta^{*}, u\right\rangle+\left\langle\lambda^{*}, v\right\rangle<0, \quad \forall(u, v) \in-(\text { qi } P) \times Q \supseteq-(S \times\{0\}),
$$

which proves (3.4).

Assume that (b) holds. By Theorem 3.5, noticing that $\overline{\text { cone }}($ co $S)=\bar{P}$ implies that $S^{*}=$ $P^{*}$,

$$
\overline{\operatorname{cone}}\left(\cos \mathcal{E}_{\bar{x}}\right) \cap-(\overline{\operatorname{cone}}(\cos S) \backslash\{0\}) \times\{0\}=\emptyset
$$

which implies

$$
\mathcal{E}_{\bar{x}} \cap-(S \backslash\{0\}) \times\{0\}=\emptyset .
$$

Since $0 \notin S$, the latter asserts that $\bar{x} \in E_{S}$ in view of Proposition 3.3.

Remark 4.2. Set $l(P):=P \cap(-P)$. The particular cases $S=$ qi $P$ and $S=P \backslash l(P)=$ $P \backslash-P$, with $P$ being a convex cone, satisfy

$$
S+P \subseteq S ; \bar{S}=\bar{P} .
$$

In fact, for the first case both properties are well-known. For the second case, we proceed as follows. Since $S+P \subseteq P$, we only need to show that $(S+P) \cap l(P)=\emptyset$. Suppose that $s+p=\ell$, for some $s \in S, p \in P, \ell \in l(P)$. Then $s=\ell-p$, i.e., $s \in S \cap(l(P)-P) \subseteq S \cap(-P)$, reaching a contradiction.

We now prove the equality. It is enough to prove that $\bar{P} \subseteq \bar{S}$, the reverse inclusion being obvious. By the previous remark, $S+P=S$, and by Lemma 3.1, $\bar{S}+\bar{P}=\bar{S}$. Noticing that $S \cup\{0\}$ is a cone then $0 \in \bar{S}$ and by the previous equality

$$
\bar{P} \subseteq \bar{S}+\bar{P}=\bar{S}
$$

which proves our assertion.

Next theorem singles out the two particular situations just mentioned.

THEOREM 4.3. Let us consider problem (1.1) with $P$ being a convex cone in $Y$ satisfying $\bar{P} \neq Y$.

(a) Let $S=$ qi $P \neq \emptyset$. If there exist $\theta^{*} \in P^{*} \backslash\{0\}$ and $\lambda^{*} \in Q^{*}$, such that $\left(\lambda^{*}, \bar{x}\right)$ is a saddle point for $L\left(\theta^{*}, \lambda, x\right)$ on $Q^{*} \times C$, then $\bar{x} \in E_{W}$. 
(b) Let $S=P \backslash l(P)$ assumed to be nonempty. If there exist $\theta^{*} \in$ qi $P^{*}$ and $\lambda^{*} \in Q^{*}$ such that $\left(\lambda^{*}, \bar{x}\right)$ is a saddle point for $L\left(\theta^{*}, \lambda, x\right)$ on $Q^{*} \times C$, then $\bar{x} \in E$.

Proof. (a) It follows from Theorem 4.1 (a) where we set $S \doteq$ qi $P$.

(b) It follows from Theorem 4.1 (b) where we set $S \doteq P \backslash l(P)$.

We have shown in the proof of Theorem 3.10 that the existence of a saddle point under the assumption (3.16) ensures that $\theta^{*} \in P^{*} \backslash\{0\}$ (note that the assumption $\overline{\operatorname{cone}}($ co $S)=\bar{P}$ in Theorem 3.10 implies $S^{*}=P^{*}$ ).

We now apply the results of the previous section by employing the convexity of the set $\overline{\text { cone }} \mathcal{E}_{\bar{x}}$, in order to avoid the presence of the convex hull in the required assumptions.

Theorem 4.4. Let $\bar{x} \in K, S=$ qri $P \neq \emptyset$, and let (3.15) be fulfilled. Moreover, assume that the following conditions hold:

(a) $\overline{\text { cone }} \mathcal{E}_{\bar{x}}$ is a convex set;

(b) $(0,0) \notin$ qri $\overline{\text { cone }} \mathcal{E}_{\bar{x}}$;

Then there exist $\theta^{*} \in P^{*} \backslash\{0\}$ and $\lambda^{*} \in Q^{*}$, such that $\left(\lambda^{*}, \bar{x}\right)$ is a saddle point for $L\left(\theta^{*}, \lambda, x\right)$ on $Q^{*} \times C$.

Proof. We preliminarly note that, since $\bar{x} \in K$, then $0 \in g(C)+Q$ and $(0,0) \in \mathcal{E}_{\bar{x}}$. By assumption $(a)$, it is easy to show that

$$
\overline{\operatorname{cone}}\left(\operatorname{co} \mathcal{E}_{\bar{x}}\right)=\overline{\text { cone }} \mathcal{E}_{\bar{x}},
$$

and, by assumption $(b), \overline{c o n e}\left(\operatorname{co} \mathcal{E}_{\bar{x}}\right)$ is not a linear subspace, or, equivalently, $(0,0) \notin$ $\operatorname{qri}\left[\operatorname{cone}\left(\cos \mathcal{E}_{\bar{x}}\right)\right]$.

By Theorem 3.9, we complete the proof. $\square$

Theorem 4.5. Let $\bar{x} \in K, S=$ qi $P \neq \emptyset$, and let (3.16) be fulfilled. Moreover, assume that the following conditions hold:

(a) $\overline{\text { cone }} \mathcal{E}_{\bar{x}}$ is a convex set;

(b) $(0,0) \notin$ qi $\overline{\text { cone }} \mathcal{E}_{\bar{x}}$;

Then there exist $\theta^{*} \in P^{*} \backslash\{0\}$ and $\lambda^{*} \in Q^{*}$, such that $\left(\lambda^{*}, \bar{x}\right)$ is a saddle point for $L\left(\theta^{*}, \lambda, x\right)$ on $Q^{*} \times C$ and, consequently, $\bar{x} \in E_{W}$.

Proof. We recall that, since $\bar{x} \in K$, then $0 \in g(C)+Q$ and $(0,0) \in \mathcal{E}_{\bar{x}}$. By assumptions (a) and (b), it follows that

$$
\overline{\mathrm{cone}}\left(\cos \mathcal{E}_{\bar{x}}\right)=\overline{\text { cone }} \mathcal{E}_{\bar{x}} \neq Y \times Z,
$$

or, equivalently, $0 \notin \mathrm{qi}\left[\operatorname{cone}\left(\operatorname{co} \mathcal{E}_{\bar{x}}\right)\right]$.

By Theorem 3.10, we obtain the first part of the thesis, while Theorem 4.3 guarantees that $\bar{x} \in E_{W} \cdot \square$

The convexity of the set $\overline{\text { cone }} \mathcal{E}_{\bar{x}}$, required by the previous theorems, can be guaranteed by employing generalized convexity assumptions on the involved functions.

Definition 4.6. (see [34, 35]) Let $X$ and $Y$ be real topological linear spaces, $C \subseteq X$, $A \subseteq Y$ a convex cone, and $f: X \rightarrow Y$ a mapping.

(i) $f$ is said to be $A$-preconvexlike on $C$, if, for any $x_{1}, x_{2} \in C$ and $\left.\alpha \in\right] 0,1[$, there exists $x_{3} \in C$ and $\rho>0$, such that:

$$
\alpha f\left(x_{1}\right)+(1-\alpha) f\left(x_{2}\right)-\rho f\left(x_{3}\right) \in A
$$


by [34], this is equivalent to cone $+f(C)+A$ convex.

(ii) $f$ is said to be generalized $A$-subconvexlike on $C$ if cone $f(C)+$ qri $A$ is convex, provided that qri $A \neq \emptyset$.

Generalized set-valued $A$-subconvexlike maps were considered in [35] in order to obtain Lagrangian type optimality conditions for a set-valued optimization problem.

REMARK 4.7. The previous convexity assumptions are among the weakest existing in the literature. Indeed, it is possible to show that if $f$ is $A$-convexlike on $C$ then it is also $A$ preconvexlike on $C$ provided that $0 \in f(C)+A$, where we recall that $f$ is said to be $A$-convexlike on $C$, if, for any $x_{1}, x_{2} \in C$ and any $\left.\alpha \in\right] 0,1\left[\right.$, there exists $x_{3} \in C$, such that:

$$
\alpha f\left(x_{1}\right)+(1-\alpha) f\left(x_{2}\right)-f\left(x_{3}\right) \in A
$$

Actually, it is known that $f$ is $A$-convexlike on $C$, if, and only if the set $f(C)+A$ is convex [30], so that the implication follows from the characterization reported in Definition $4.6(i)$. Similarly, if qri $A \neq \emptyset$, it can be shown that if $f$ is $A$-preconvexlike on $C$, then it is generalized $A$-subconvexlike on $C$.

Given $\bar{x} \in C$, let $G_{\bar{x}}(x): X \rightarrow Y \times Z$ be the function defined by

$$
G_{\bar{x}}(x):=(f(x)-f(\bar{x}), g(x)) .
$$

Corollary 4.8. Let $\bar{x} \in K, S=$ qi $P \neq \emptyset$, and let (3.15) and assumption (b) of Theorem 4.4 be fulfilled. Moreover, suppose that any of the following conditions hold:

$\left(a^{\prime}\right) G_{\bar{x}}$ is generalized $(P \times Q)$-subconvexlike on $C$, provided that qri $Q \neq \emptyset$;

$\left(a^{\prime \prime}\right) G_{\bar{x}}$ is $(P \times Q)$-preconvexlike on $C$.

Then, there exist $\theta^{*} \in P^{*} \backslash\{0\}$ and $\lambda^{*} \in Q^{*}$, such that $\left(\lambda^{*}, \bar{x}\right)$ is a saddle point for $L\left(\theta^{*}, \lambda, x\right)$ on $Q^{*} \times C$ and $\bar{x} \in E_{W}$.

Proof. It is enough to prove that $\left(a^{\prime}\right)$ or $\left(a^{\prime \prime}\right)$ imply assumption $(a)$ of Theorem 4.4. Suppose that $\left(a^{\prime}\right)$ holds. Note that

$$
\overline{\operatorname{cone}\left(G_{\bar{x}}(C)\right)+(P \times Q)}=\overline{\text { cone }} \mathcal{E}_{\bar{x}} \text { and } \operatorname{qri}(P \times Q) \neq \emptyset
$$

Since $\overline{M+N}=\overline{M+\bar{N}}=\overline{M+\overline{\text { qri } N}}$ for all convex set $N$ such that qri $N \neq \emptyset$, we get

$$
\overline{\operatorname{cone}\left(G_{\bar{x}}(C)\right)+\operatorname{qri}(P \times Q)}=\overline{\text { cone }} \mathcal{E}_{\bar{x}} .
$$

Taking into account $(4.2),\left(a^{\prime}\right)$ implies the convexity of $\overline{\text { cone }} \mathcal{E}_{\bar{x}}$.

Suppose that $\left(a^{\prime \prime}\right)$ holds. By Definition 4.6 (i) where we set $f:=G_{\bar{x}}$ and $A:=P \times Q$ it follows that cone $_{+} \mathcal{E}_{\bar{x}}$ is a convex set. This implies that cone $\mathcal{E}_{\bar{x}}$ is a convex set so that $\overline{c o n e} \mathcal{E}_{\bar{x}}$ is convex.

In the next result, (see also [29]), we assume that the topological interiors of the cones $P$ and $Q$ are nonempty. In such a case the (weak) optimality of $\bar{x}$ guarantees that assumption $(b)$ of Theorem 4.5 is fulfilled.

Corollary 4.9. Let $\bar{x} \in C$ and assume that the following conditions hold:

(a) $S=\operatorname{int} P \neq \emptyset$ and int $Q \neq \emptyset$; 
(b) $0 \in \operatorname{cog} g(C)+\operatorname{int} Q$.

If additionally $G_{\bar{x}}$ is generalized $(P \times Q)$-subconvexlike on $C$, then

$$
\bar{x} \in E_{W} \Longleftrightarrow\left\{\begin{array}{c}
\exists\left(\theta^{*}, \lambda^{*}\right) \in\left(P^{*} \backslash\{0\}\right) \times Q^{*}: \bar{x} \in \underset{C}{\operatorname{argmin}} L\left(\theta^{*}, \lambda^{*}, \cdot\right) \\
\left\langle\lambda^{*}, g(\bar{x})\right\rangle=0, g(\bar{x}) \in-Q .
\end{array}\right.
$$

Proof. (If): It follows from Theorem 4.3.

(Only if): It is enough to show that the assumptions of Theorem 4.5 are fulfilled. We preliminarly note that, by assumption $(a),(3.16)$ is equivalent to assumption $(b)$ and, as in the proof of Corollary 4.8, $\left(a^{\prime}\right)$ implies that (a) of Theorem 4.5 holds.

By Proposition 3.3, $\bar{x} \in E_{W}$ iff (3.4) holds with $S:=$ qri $P=$ int $P \neq \emptyset$, or, equivalently,

$$
\text { cone } \mathcal{E}_{\bar{x}} \cap-[(\text { int } P) \times Q]=\emptyset \text {. }
$$

Since int $Q \neq \emptyset$, then $\operatorname{int}[($ int $P) \times Q]=\operatorname{int}(P \times Q) \neq \emptyset$, which implies, together with (4.3) that cone $\mathcal{E}_{\bar{x}} \neq Y \times Z$, i.e., $(b)$ of Theorem 4.5 is fulfilled, which completes the proof.

When the image space associated with (1.1) is finite dimensional (i.e., when $Y$ and $Z$ are finite dimensional), the weak optimality of $\bar{x}$ implies assumption (b) of Theorem 4.4 even if $P$ has empty interior.

Corollary 4.10. Let $\bar{x} \in E_{W}$, let $P$ be a closed convex cone with $0 \notin$ qri $P$, and assume that the following conditions hold:

$\left(a^{\prime \prime}\right) G_{\bar{x}}$ is $(P \times Q)$-preconvexlike on $C$;

$\left(b^{\prime \prime}\right) Y$ and $Z$ are finite dimensional spaces;

$\left(c^{\prime \prime}\right) 0 \in \operatorname{ri}[\operatorname{cone}(g(C)+Q)]$.

Then, there exist $\theta^{*} \in P^{*} \backslash\{0\}$ and $\lambda^{*} \in Q^{*}$, such that $\left(\lambda^{*}, \bar{x}\right)$ is a saddle point for $L\left(\theta^{*}, \lambda, x\right)$ on $Q^{*} \times C$.

Proof. It is enough to prove that the assumptions of Theorem 4.4 are fulfilled.

We preliminarly recall that, for a nonempty convex subset $M$ of a finite dimensional t.v.s., qri $M=$ ri $M=$ ri $\bar{M}$ (see, e.g., [5, 31]). Noticing that, by $\left(a^{\prime \prime}\right)$, $\operatorname{cone}(g(C)+Q)$ is a convex set, by Lemma 2.1 (a) it follows that $\left(c^{\prime \prime}\right)$ is equivalent to $0 \in \operatorname{ri}[\operatorname{co}(g(C)+Q)]$ i.e., to (3.15).

As shown in the proofs of Corollaries 4.8 and 4.9 , cone $\mathcal{E}_{\bar{x}}$ is a convex set and condition $(a)$ of Theorem 4.4 is fulfilled. Moreover, since $\bar{x} \in E_{W}$, then, by Proposition 3.3 with $S=$ qri $P=$ ri $P$,

$$
\text { cone } \mathcal{E}_{\bar{x}} \cap-[(\text { ri } P) \times\{0\}]=\emptyset .
$$

This yields,

$$
\begin{aligned}
& (0,0) \notin\left[\operatorname{ri}\left(\text { cone } \mathcal{E}_{\bar{x}}\right)+(\operatorname{ri}(P) \times\{0\})\right]=\operatorname{ri}\left(\text { cone } \mathcal{E}_{\bar{x}}\right)+\operatorname{ri}(P \times\{0\}) \\
= & \operatorname{ri}\left(\text { cone } \mathcal{E}_{\bar{x}}+(P \times\{0\})\right)=\operatorname{ri} \text { cone }\left(\mathcal{E}_{\bar{x}}+(P \times\{0\})\right)=\operatorname{ri}\left(\text { cone } \mathcal{E}_{\bar{x}}\right) .
\end{aligned}
$$

Therefore condition (b) of Theorem 4.4 is fulfilled, which completes the proof. 
5. Applications to bicriteria problems: proper efficiency, efficiency and weak efficiency. In this section, we deal with (Benson) proper efficient, efficient and weak efficient solutions to a general bicriteria optimization problem with geometric constraints. In the first subsection we consider geometric characterizations in the image space of the possibility of obtaining proper and weak efficiency by means of a linear scalarization, while in the second subsection the same characterizations are expressed in the given space by means of level sets of the objective functions. The presented results supplement those established in Sections 2.5.1 and 2.5.2 in [13].

We point out that the underlying (not necessarily closed) convex cone $P$ has possibly empty interior.

Next proposition, taken from [13] after a careful reading of its proof (where the assumption $A \cap(-$ ri $P)=\emptyset$ was forgotten), will play an important role in subsequent sections.

Proposition 5.1. [13, Theorem 2.4] Let $P \subseteq \mathbb{R}^{2}$ be a nontrivial convex cone, $\emptyset \neq A \subseteq \mathbb{R}^{2}$ such that $A \cap(-$ ri $P)=\emptyset$ and

$$
\operatorname{int}(\overline{\operatorname{cone}}(A+P)) \neq \emptyset
$$

The following assertions are equivalent:

(a) $0 \notin \operatorname{int}(\overline{\operatorname{cone}}(A+P))$ and $\overline{\operatorname{cone}}(A+P)$ is convex;

(b) $\exists p^{*} \in P^{*} \backslash\{0\}$ such that $\left\langle p^{*}, a\right\rangle \geq 0 \quad \forall a \in A$.

In case $0 \in A$, they are also equivalent to

(c) $\operatorname{cone}(A+P)$ is convex.

Notice that when int $P \neq \emptyset$ condition (5.1) is superfluous.

Proof. $(a) \Rightarrow(b)$ : It is a consequence of a simple result of separation of convex sets.

$(b) \Rightarrow(a) ;(b) \Rightarrow(c)$ : Let $L \doteq\left\{x \in \mathbb{R}^{2}:\left\langle p^{*}, x\right\rangle \geq 0\right\}$, then $\overline{\operatorname{cone}}(A+P) \subseteq L$, implying the first part of $(a)$. We now observe that

$$
x \in A, 0 \neq p \in \operatorname{ri} P, A \cap(- \text { ri } P)=\emptyset \Longrightarrow\left\{\begin{array}{l}
\text { either } x=t p, t \geq 0, \text { or } \\
\{x, p\} \text { is linearly independent. }
\end{array}\right.
$$

Choose $p \in \operatorname{ri} P \backslash\{0\}$ and let $y, z \in A, y \neq z$; then

$$
\operatorname{cone}\{y\}+\operatorname{cone}\{p\}=\{\lambda y+\mu p: \lambda, \mu \geq 0\}
$$

is a closed convex cone containing $y$ and $p$ and contained in $\overline{\operatorname{cone}}(A+P)$. The same is true for cone $\{z\}+$ cone $\{p\}$. Since for $x=y$ or $x=z,\{p, x\}$ is linearly independent, or $p=t x$, with $t>0$, because of $(5.2)$, then the two cones are pointed and have the halfline $\{t p, t \geq 0\}$ in common and are contained in $\overline{\operatorname{cone}}(A+P) \subseteq L$. Hence the set

$$
B \doteq(\operatorname{cone}\{y\}+\operatorname{cone}\{p\}) \cup(\operatorname{cone}\{z\}+\operatorname{cone}\{p\})
$$

is a convex cone. Since $y, z \in B$, then $[y, z] \subseteq B \subseteq \operatorname{cone}(A+P)$. Thus $\operatorname{co}(A) \subseteq \operatorname{cone}(A+P)$. Then,

$$
\operatorname{co}(A+P)=\operatorname{co}(A)+P \subseteq \overline{\operatorname{cone}}(A+P)+P \subseteq \overline{\operatorname{cone}(A+P)+P} \subseteq \overline{\operatorname{cone}}(A+P)
$$


yielding $\overline{\operatorname{cone}}(\operatorname{co}(A+P)) \subseteq \overline{\operatorname{cone}}(A+P)$. The reverse inclusion is obvious, and therefore $\overline{\operatorname{cone}}(A+$ $P)$ is convex.

In case $0 \in A$, we proceed as follows. We have

$$
\operatorname{co}(A+P)=\operatorname{co}(A)+P \subseteq \operatorname{cone}(A+P)+P=\operatorname{cone}(A+P)
$$

yielding cone $(\operatorname{co}(A+P)) \subseteq \operatorname{cone}(A+P)$. The reverse inclusion is obvious and so cone $(A+P)$ is convex.

$(c) \Rightarrow(b)$ : It follows from a simple result of separation of convex sets, since

$$
A \cap(- \text { ri } P)=\emptyset \Leftrightarrow \operatorname{cone}(A+P) \cap(- \text { ri } P)=\emptyset,
$$

which trivially holds in case int $P \neq \emptyset$; and when int $P=\emptyset$, we observe that $\operatorname{dim} P=1$ and $P$ is pointed because of $0 \in A$, and then $0 \notin$ ri $P$, and hence the equivalence is satisfied. $\square$

REMARK 5.2. Looking at the proof carefully, one may realize that assumption (5.1) can be deleted, but instead (a) is substituted by

$$
\overline{\operatorname{cone}}(A+P) \neq \mathbb{R}^{2} \text { and } \overline{\operatorname{cone}}(A+P) \text { is convex. }
$$

Next, the case

$$
\operatorname{int}\left(\operatorname{cone}_{+}(A+P)\right)=\emptyset
$$

is considered.

Proposition 5.3. Let $P \subseteq \mathbb{R}^{2}$ be a nontrivial convex cone, $\emptyset \neq A \subseteq \mathbb{R}^{2}$. The following assertions hold:

(a) $\operatorname{int}\left(\operatorname{cone}_{+}(A+P)\right)=\emptyset \Longleftrightarrow \operatorname{int}(\operatorname{cone}(A+P))=\emptyset$.

(b) If $\operatorname{int}(\operatorname{cone}(A+P))=\emptyset$ then $\operatorname{dim} P=1, P$ is closed and either

$$
\operatorname{cone}(A+P)=P, \text { or cone }(A+P)=P \cup(-P) .
$$

Consequently, cone $(A+P)$ is convex and $\overline{\operatorname{cone}}(A+P)=\operatorname{cone}(A+P)$, and therefore

$$
\operatorname{int}(\operatorname{cone}(A+P))=\emptyset \Longleftrightarrow \operatorname{int}(\overline{\operatorname{cone}}(A+P))=\emptyset \text {. }
$$

Proof. (a): The "only if" part is direct. For the "if" part, suppose that $U \subseteq \operatorname{cone}(A+P)$ for some open set $U$. Then, $U \backslash\{0\} \subseteq$ cone $_{+}(A+P)$, implying that $\operatorname{int}\left(\right.$ cone $\left._{+}(A+P)\right) \neq \emptyset$, a contradiction.

(b): Obviously $\operatorname{dim} P=1$ and $P$ is closed. Assume that $\left\{x, p_{0}\right\}$ is linearly independent for some $x \in A$ and some $p_{0} \in P$. Then $\operatorname{int}(\operatorname{cone}(x+P)) \neq \emptyset$, and since cone $(x+P) \subseteq \operatorname{cone}(A+P)$, we reach a contradiction.

Hence $\{x, p\}$ is linearly dependent for all $x \in A$ and all $p \in P$. This yields either cone $(A+P)=P$ or cone $(A+P)=P \cup(-P)$, and the proof is complete. $\square$

Next proposition considers the case where $P$ is pointed and

$$
\operatorname{int}\left[\operatorname{cone}_{+}(f(C)-f(\bar{x})+P)\right]=\emptyset .
$$

Proposition 5.4. Let $f, C$ be as above, with $P=\mathbb{R}_{+} d, d \neq 0$, and $\bar{x} \in C$. The following hold: 
(a) If cone $(f(C)-f(\bar{x})+P)=P$ then $\bar{x} \in E_{i}$ (put $S=\mathbb{R}^{2} \backslash-P$ in (1.1)), and so $\bar{x} \in E_{p r}$.

(b) If cone $(f(C)-f(\bar{x})+P)=P \cup(-P)$ then $\bar{x} \notin E_{W}$, (put $S=$ ri $P$ in (1.1)).

Proof. It is a consequence of Proposition 5.3.

Furthermore, it is possible to prove the following result.

Proposition 5.5. Let $P \subseteq \mathbb{R}^{2}$ be a nontrivial convex cone. Consider the next statements:

(a) $\bar{x} \in E_{W}(S=$ ri $P)$ and $\overline{\operatorname{cone}}(f(C)-f(\bar{x})+P)$ is nonconvex;

(b) $\bar{x} \in E_{W}$, int $\left(\right.$ cone $\left._{+}(f(C)-f(\bar{x})+P)\right) \neq \emptyset$ and

$$
\operatorname{cone}(\operatorname{co} f(C)-f(\bar{x})+P)=\mathbb{R}^{2} .
$$

(c) $\bar{x} \in E_{W}$ and cone $(f(C)-f(\bar{x})+P)$ is nonconvex.

Then,

$$
(a) \Longrightarrow(b) \Longleftrightarrow(c)
$$

In case int $P \neq \emptyset,(a)$ and $(b)$ are equivalent.

Proof. $(a) \Rightarrow(b)$ : If $\operatorname{int}\left(\right.$ cone $\left._{+}(f(C)-f(\bar{x})+P)\right)=\emptyset$ then by Proposition 5.3 applied to $A=f(C)-f(\bar{x})$, we get the convexity of cone $(f(C)-f(\bar{x})+P)$, a contradiction.

Now suppose that cone $(\operatorname{co} f(C)-f(\bar{x})+P) \neq \mathbb{R}^{2}$. Then

$$
0 \notin \operatorname{int}(\operatorname{co} f(C)-f(\bar{x})+P),
$$

which implies, via a separation result, the existence of $p^{*} \in P^{*} \backslash\{0\}$ such that $\left\langle p^{*}, \xi\right\rangle \geq 0$ for all $\xi \in f(C)-f(\bar{x})$. This together with the fact that $\bar{x} \in E_{W}$, that is, $(f(C)-f(\bar{x})) \cap(-$ ri $P)=\emptyset$, Proposition 5.1 (applied to $A=f(C)-f(\bar{x})$ ) allows us to conclude that cone $(f(C)-f(\bar{x})+P)$ is convex, a contradiction again.

$(b) \Rightarrow(c)$ : If cone $(f(C)-f(\bar{x})+P)$ is convex, then we know that

$$
\text { cone }(\operatorname{co} f(C)-f(\bar{x})+P)=\operatorname{cone}(f(C)-f(\bar{x})+P),
$$

and therefore cone $(f(C)-f(\bar{x})+P)=\mathbb{R}^{2}$, which is impossible if $\bar{x} \in E_{W}$.

$(c) \Rightarrow(b)$ : The nonemptiness of $\operatorname{int}\left(\right.$ cone $\left._{+}(f(C)-f(\bar{x})+P)\right)$ is obtained by applying Proposition 5.3. To prove the second part, suppose to the contrary that cone $($ co $f(C)-f(\bar{x})+P) \neq \mathbb{R}^{2}$. Then $0 \notin \operatorname{int}(\operatorname{co} f(C)-f(\bar{x})+P)$, and so by a separation theorem, we get the existence of $p^{*} \in P^{*} \backslash\{0\}$ such that $\left\langle p^{*}, \xi\right\rangle \geq 0$ for all $\xi \in f(C)-f(\bar{x})+P$. Noticing that $0 \in f(C)-f(\bar{x})$, by Proposition 5.1 the convexity of $\operatorname{cone}(f(C)-f(\bar{x})+P)$ follows, reaching a contradiction. The fact $(b) \Rightarrow(a)$ follows from the equivalence:

$$
\bar{x} \in E_{W} \Longleftrightarrow \overline{\operatorname{cone}}(f(C)-f(\bar{x})+P) \cap(- \text { int } P)=\emptyset,
$$

and so the proof is completed.

Example 5.11 shows that $(c)$ does not imply $(a)$.

5.1. Geometric characterization of efficiency. We particularize Theorem 3.6 to the case $Y=\mathbb{R}^{2}$ :

TheOrem 5.6. Assume that $S \subseteq \mathbb{R}^{2}$ is a nonempty set and $S+P \subseteq S$ with $P$ being a convex cone. The following assertions are equivalent: 
(a) int $S^{*} \neq \emptyset$ and $\bar{x} \in \bigcup_{\theta^{*} \in \operatorname{int} S^{*}} \underset{C}{\operatorname{argmin}}\left\langle\theta^{*}, f(\cdot)\right\rangle$;

(b) $\bar{x} \in C, \overline{\operatorname{cone}}(\operatorname{co} S)$ is pointed and

$$
\overline{\operatorname{cone}}(\cos f(C)-f(\bar{x})+P) \cap(-\overline{\operatorname{cone}}(\cos S) \backslash\{(0,0)\})=\emptyset .
$$

If additionally $\overline{\text { cone }}($ co $S)=\bar{P}$ (in particular if $S=P$ ) the previous result reveals a hidden convexity of some extended image set of $f$, as a consequence of Proposition 5.1, as next corollary shows.

Corollary 5.7. Let $P \subseteq \mathbb{R}^{2}$ be a convex cone (having possibly empty interior). Then $(a)$ and $(b)$ are equivalent:

(a) int $P^{*} \neq \emptyset$ and $\bar{x} \in \bigcup_{\theta^{*} \in \operatorname{int} P^{*}}^{\operatorname{argmin}}\left\langle\theta^{*}, f(\cdot)\right\rangle$;

(b) $\bar{x} \in C$ and $\overline{\text { cone }}(\cos f(C)-f(\bar{x})+P) \cap(-\bar{P})=\{(0,0)\}$.

Moreover, if additionally $\operatorname{int}[\overline{\operatorname{cone}}(f(C)-f(\bar{x})+P)] \neq \emptyset$, then any of $(a)$ or $(b)$ is equivalent to

(c) $\bar{x} \in C$, $\overline{\mathrm{cone}}(f(C)-f(\bar{x})+P) \cap(-\bar{P})=\{(0,0)\}$ and $\overline{\mathrm{cone}}(f(C)-f(\bar{x})+P)$ is convex.

Proof. The equivalence between $(a)$ and $(b)$ follows directly from Theorem 5.6 setting $S \doteq P$ and noticing that $(b)$ implies that $\bar{P}$ is pointed.

$(c) \Rightarrow(b)$ : Set $\mathcal{E}_{0 \bar{x}}:=f(C)-f(\bar{x})+P$. The result follows since $\overline{\text { cone }}\left(\right.$ co $\left.\mathcal{E}_{0 \bar{x}}\right)=\overline{\text { cone }} \mathcal{E}_{0 \bar{x}}$, provided that $\overline{\text { cone }} \mathcal{E}_{0 \bar{x}}$ is convex, as noticed in the previous section.

$(a) \Rightarrow(c)$ : Assume that int $[\overline{\operatorname{cone}}(f(C)-f(\bar{x})+P)] \neq \emptyset$. Then, $(a)$ implies that $(b)$ of Proposition 5.1 and $(f(C)-f(\bar{x})) \cap(-$ ri $P)=\emptyset$ are satisfied. Indeed, if there exists $x_{0} \in C$ such that $f\left(x_{0}\right)-f(\bar{x}) \in-$ ri $P$, one gets $f\left(x_{0}\right)-f(\bar{x}) \neq 0$ (due to pointedness of $P$ ) and so $\left\langle\theta^{*}, f\left(x_{0}\right)-\right.$ $f(\bar{x})\rangle<0$, a contradiction with $(a)$. Hence we can apply Proposition 5.1 to infer that

$$
\overline{\text { cone }}(f(C)-f(\bar{x})+P) \text { is convex, }
$$

which completes the proof.

In what follows, a characterization for a point, being a (Benson) proper efficient solution, to be also a minimizer of a linear scalarization of the bicriteria optimization problem, is established.

TheOREM 5.8. Let $P \subseteq \mathbb{R}^{2}$ be a closed convex cone (having possibly empty interior). The following assertions are equivalent:

(a) int $P^{*} \neq \emptyset$ and $\bar{x} \in \bigcup_{\theta^{*} \in \text { int } P^{*}} \underset{C}{\operatorname{argmin}}\left\langle\theta^{*}, f(\cdot)\right\rangle$;

(b) $\bar{x} \in E_{p r}$ and $\overline{\operatorname{cone}}(f(C)-f(\bar{x})+P)$ is convex.

Proof. (b) implies (a) always holds, since $\bar{x} \in E_{p r}$ implies the pointedness of $P$, and by Theorem 5.6, the assertion follows. As regards $(a)$ implies $(b)$, by Corollary 5.7 we have $\bar{x} \in E_{p r}$ which implies $\overline{\text { cone }}(f(C)-f(\bar{x})+P) \neq Y$. Moreover, by assumption $(a)$ it follows that $(b)$ of Proposition 5.1 holds with $A=f(C)-f(\bar{x})$, which implies that $\overline{\operatorname{cone}}(f(C)-f(\bar{x})+P)$ is convex, taking into account Remark 5.2.

We now deal with weak efficient solutions. On this regards, the following result, which is a consequence of Proposition 5.1, is obtained.

TheOREM 5.9. Let $P \subseteq \mathbb{R}^{2}$ be a convex cone with int $P \neq \emptyset$. The following assertions are equivalent: 
(a) $\bar{x} \in \bigcup_{\theta^{*} \in P^{*} \backslash\{0\}} \underset{C}{\operatorname{argmin}}\left\langle\theta^{*}, f(\cdot)\right\rangle$;

(b) $\bar{x} \in E_{W}$ and $\overline{\operatorname{cone}}(f(C)-f(\bar{x})+P)$ is convex.

The following examples show that the previous equivalence does not hold if int $P$ is substituted by ri $P$ when int $P=\emptyset$.

ExAmple 5.10. Let $f_{i}: \mathbb{R} \rightarrow \mathbb{R}, i=1,2$, defined by $f_{1}(x)=x^{3}, f_{2}(x)=x^{2}-1$. Let $P=\mathbb{R}_{+}(1,0), C=\{0,1,-1\}$ and $\bar{x}=1$. Then

$$
f(C)-f(\bar{x})=\{(-1,-1),(0,0),(-2,0)\} .
$$

Let $\theta^{*}=(0,-1) \in P^{*}$. It is simple to see that $\bar{x}$ is a minimum for $\left\langle\theta^{*}, f(x)\right\rangle=1-x^{2}$ on $C$ but $\bar{x} \notin E_{W}$ since $(f(C)-f(\bar{x})) \cap(-$ ri $P) \neq \emptyset$. Therefore $(a)$ does not imply $(b)$.

Example 5.11. Let $f_{i}: \mathbb{R} \rightarrow \mathbb{R}, i=1,2$, defined by $f_{1}(x)=-x^{2}, f_{2}(x)=x^{3}$. Let $P=\mathbb{R}_{+}(1,0), C=\mathbb{R}$ and $\bar{x}=0$. Then

$$
f(C)-f(\bar{x})=\left\{(u, v) \in \mathbb{R}^{2}: u=-x^{2}, v=x^{3}, x \in \mathbb{R}\right\}=\left\{(u, v) \in \mathbb{R}^{2}: u=-v^{\frac{2}{3}}, v \in \mathbb{R}\right\}
$$

yielding $\overline{c o n e}(f(C)-f(\bar{x})+P)=\mathbb{R}^{2}$, so that (b) holds. Since $P^{*}=\mathbb{R}_{+} \times \mathbb{R}$, then

$$
\min _{x \in C}\left\langle p^{*}, f(x)\right\rangle=-\infty, \quad \forall p^{*} \in P^{*},
$$

and therefore (b) does not imply (a).

It is of interest to establish conditions which ensure the convexity of the set $\overline{\operatorname{cone}}(f(C)-$ $f(\bar{x})+P)$. A standard situation where this happens is the quadratic homogeneous case, i.e., $f(x):=\left(x^{\top} A_{1} x, x^{\top} A_{2} x\right)$ with $A_{1}, A_{2}$ symmetric matrices and $C \doteq \mathbb{R}^{n}$. In such a case $f\left(\mathbb{R}^{n}\right)$ is a convex set [12] which yields that $\overline{\text { cone }} \mathcal{E}_{0 \bar{x}}$ is convex. More generally, consider $f=\left(f_{1}, f_{2}\right)$ where $f_{i}(x)=\frac{1}{2} x^{\top} A_{i} x+a_{i}^{\top} x+\alpha_{i}$, where $A_{i}^{\top}=A_{i}, a_{i} \in \mathbb{R}^{n}, \alpha_{i} \in \mathbb{R}$. Then, by Theorem 4.18 in [17], $f(C)+\mathbb{R}_{+}^{2}$ is convex provided $C=\left\{x \in \mathbb{R}^{n}: H x=d\right\}$ is any affine space.

5.2. Characterization of efficiency in terms of level sets. Set $P=\mathbb{R}_{+}^{2}$ and $\bar{x} \in E_{W}$. We aim now at characterizing the set cone $\left(\operatorname{co} f(C)-f(\bar{x})+\mathbb{R}_{+}^{2}\right)$ according to the properties of suitable level sets of the components of the objective function defined as follows. For $i=1,2$, set

$$
\begin{gathered}
S_{i}^{-}(\bar{x}):=\left\{x \in K: f_{i}(x)<f_{i}(\bar{x})\right\} ; \quad S_{i}^{+}(\bar{x}):=\left\{x \in K: f_{i}(x)>f_{i}(\bar{x})\right\} ; \\
S_{i}^{=}(\bar{x}):=\left\{x \in K: f_{i}(x)=f_{i}(\bar{x})\right\} .
\end{gathered}
$$

Whenever $S_{1}^{+}(\bar{x}) \cap S_{2}^{-}(\bar{x}) \neq \emptyset$ and $S_{1}^{-}(\bar{x}) \cap S_{2}^{+}(\bar{x}) \neq \emptyset$, we set

$$
\alpha:=\inf _{x \in S_{1}^{+}(\bar{x}) \cap S_{2}^{-}(\bar{x})} \frac{f_{2}(x)-f_{2}(\bar{x})}{f_{1}(x)-f_{1}(\bar{x})}, \quad \beta:=\sup _{x \in S_{1}^{-}(\bar{x}) \cap S_{2}^{+}(\bar{x})} \frac{f_{2}(x)-f_{2}(\bar{x})}{f_{1}(x)-f_{1}(\bar{x})} .
$$

Note that $-\infty \leq \alpha<0$ and $-\infty<\beta \leq 0$.

Using the notation introduced above, we can completely describe the set

$$
\overline{\operatorname{cone}}\left(\operatorname{co}\left(f(C)-f(\bar{x})+\mathbb{R}_{+}^{2}\right)\right)=\overline{\operatorname{cone}}\left(\operatorname{co} f(C)-f(\bar{x})+\mathbb{R}_{+}^{2}\right)
$$


(see also Sections 2.5.1 and 2.5.2 in [13]). To this aim, we recall that

$$
\overline{\operatorname{cone}}\left(\operatorname{co} f(C)-f(\bar{x})+\mathbb{R}_{+}^{2}\right)=\overline{\operatorname{cone}}\left(f(C)-f(\bar{x})+\mathbb{R}_{+}^{2}\right)
$$

provided that $\overline{\operatorname{cone}}\left(f(C)-f(\bar{x})+\mathbb{R}_{+}^{2}\right)$ is convex, as already observed in the previous section.

By Proposition 5.5, it will be enough to characterize the cases where $\overline{\operatorname{cone}}\left(f(C)-f(\bar{x})+\mathbb{R}_{+}^{2}\right)$ is convex.

Notice that

$$
\begin{aligned}
& S_{1}^{-}(\bar{x}) \neq \emptyset \Leftrightarrow[f(C)-f(\bar{x})] \cap\left\{(u, v) \in \mathbb{R}^{2}: u<0\right\} \neq \emptyset, \\
& S_{2}^{-}(\bar{x}) \neq \emptyset \Leftrightarrow[f(C)-f(\bar{x})] \cap\left\{(u, v) \in \mathbb{R}^{2}: v<0\right\} \neq \emptyset,
\end{aligned}
$$

and similarly for $S_{i}^{+}(\bar{x})$ and $S_{i}^{=}(\bar{x}), i=1,2$.

The previous equivalences yield:

(a) $S_{1}^{-}(\bar{x})=\emptyset \Leftrightarrow\left[f(C)-f(\bar{x})+\mathbb{R}_{+}^{2}\right] \cap\left\{(u, v) \in \mathbb{R}^{2}: u<0\right\}=\emptyset$,

(b) $S_{2}^{-}(\bar{x})=\emptyset \Leftrightarrow\left[f(C)-f(\bar{x})+\mathbb{R}_{+}^{2}\right] \cap\left\{(u, v) \in \mathbb{R}^{2}: v<0\right\}=\emptyset$,

By (5.3) and the previous relations we obtain the description stated in the figure, where we set $\mathcal{E}_{0 \bar{x}}:=f(C)-f(\bar{x})+\mathbb{R}_{+}^{2}$.

More in details, we have the following cases:

(1) $S_{1}^{-}(\bar{x}) \cap S_{2}^{=}(\bar{x}) \neq \emptyset$ and $S_{2}^{-}(\bar{x})=\emptyset$.

Notice that in this case we have $v \geq 0, \forall(u, v) \in \mathcal{E}_{0 \bar{x}}$ and there exists $(\tilde{u}, \tilde{v}) \in \mathcal{E}_{0 \bar{x}}$ with $\tilde{u}<0, \tilde{v}=0$, which implies cone $\mathcal{E}_{0 \bar{x}}=\left\{(u, v) \in \mathbb{R}^{2}: v \geq 0\right\}$

(2) $S_{1}^{-}(\bar{x}) \cap S_{2}^{+}(\bar{x}) \neq \emptyset, \beta=0$ and $S_{2}^{-}(\bar{x})=\emptyset$.

We see that the second condition yields $v \geq 0, \forall(u, v) \in \mathcal{E}_{0 \bar{x}}$, while the first one implies that there exists $\left(u_{n}, v_{n}\right) \in \mathcal{E}_{0 \bar{x}}$, with $u_{n}<0$ such that $v_{n} \rightarrow 0$, which implies $\overline{\text { cone }} \mathcal{E}_{0 \bar{x}}=\left\{(u, v) \in \mathbb{R}^{2}: v \geq 0\right\}$.

(3) $S_{1}^{-}(\bar{x}) \cap S_{2}^{=}(\bar{x})=\emptyset, S_{1}^{-}(\bar{x}) \cap S_{2}^{+}(\bar{x}) \neq \emptyset, \beta<0$ and $S_{2}^{-}(\bar{x})=\emptyset$.

As in case $(1)$, we have $v \geq 0, \forall(u, v) \in \mathcal{E}_{0 \bar{x}}$, while

$$
S_{1}^{-}(\bar{x}) \cap S_{2}^{+}(\bar{x}) \neq \emptyset, \Leftrightarrow \sup _{\substack{(u, v) \in \mathcal{K} \\ u<0, v>0}} \frac{u}{v}=\beta \Leftrightarrow v \geq \beta u, \forall(u, v) \in \mathcal{K},
$$

where we have set $\mathcal{K}:=f(C)-f(\bar{x})$, yielding $\overline{\text { cone }} \mathcal{E}_{0 \bar{x}}=\left\{(u, v) \in \mathbb{R}^{2}: v \geq \beta u, v \geq 0\right\}$, $\beta<0$.

(4) $S_{1}^{-}(\bar{x})=\emptyset, S_{1}^{+}(\bar{x}) \cap S_{2}^{-}(\bar{x}) \neq \emptyset$ and $\alpha>-\infty$.

Notice that $S_{1}^{-}(\bar{x})=\emptyset$ implies $u \geq 0, \forall(u, v) \in \mathcal{E}_{0 \bar{x}}$, while

$$
S_{1}^{+}(\bar{x}) \cap S_{2}^{-}(\bar{x}) \neq \emptyset, \Leftrightarrow \inf _{\substack{(u, v) \in \mathcal{K} \\ u>0, v<0}} \frac{u}{v}=\alpha \Leftrightarrow v \geq \alpha u, \forall(u, v) \in \mathcal{K},
$$

yielding cone $\mathcal{E}_{0 \bar{x}}=\left\{(u, v) \in \mathbb{R}^{2}: v \geq \alpha u, u \geq 0\right\}$.

(5) $\left[S_{1}^{-}(\bar{x}) \cap S_{2}^{=}(\bar{x})=\emptyset, S_{1}^{-}(\bar{x}) \cap S_{2}^{+}(\bar{x}) \neq \emptyset, \beta<0, S_{1}^{=}(\bar{x}) \cap S_{2}^{-}(\bar{x})=\emptyset, S_{1}^{+}(\bar{x}) \cap S_{2}^{-}(\bar{x}) \neq\right.$ $\emptyset, \alpha \geq \beta$ and $\alpha>-\infty]$

Combining the results obtained in (3) and (4) we have

$$
\overline{\text { cone }} \mathcal{E}_{0 \bar{x}}=\left\{(u, v) \in \mathbb{R}^{2}: v \geq \alpha u, v \geq \beta u\right\}, \alpha \geq \beta \text {. }
$$


(6) $\left[S_{1}^{-}(\bar{x})=S_{2}^{-}(\bar{x})=\emptyset\right]$. In this case we immediately obtain

$$
\text { cone } \mathcal{E}_{0 \bar{x}}=\left\{(u, v) \in \mathbb{R}^{2}: u \geq 0, v \geq 0\right\} .
$$

(7) $S_{1}^{-}(\bar{x})=\emptyset$ and $S_{1}^{=}(\bar{x}) \cap S_{2}^{-}(\bar{x}) \neq \emptyset$.

Notice that $S_{1}^{-}(\bar{x})=\emptyset$ implies $u \geq 0, \forall(u, v) \in \mathcal{E}_{0 \bar{x}}$, while the second relation guarantees the existence of $(0, \tilde{v}) \in \mathcal{E}_{0 \bar{x}}$, with $\bar{v}<0$ yielding

$$
\overline{\text { cone }} \mathcal{E}_{0 \bar{x}}=\left\{(u, v) \in \mathbb{R}^{2}: u \geq 0\right\} .
$$

(8) $S_{1}^{-}(\bar{x})=\emptyset$ and $S_{1}^{+}(\bar{x}) \cap S_{2}^{-}(\bar{x}) \neq \emptyset$ and $\alpha=-\infty$.

Taking the limit in (4) for $\alpha \rightarrow-\infty$ we obtain

$$
\overline{\text { cone }} \mathcal{E}_{0 \bar{x}}=\left\{(u, v) \in \mathbb{R}^{2}: u \geq 0\right\} .
$$

In all the other cases not considered in the figure, $\overline{\operatorname{cone}}\left(f(C)-f(\bar{x})+\mathbb{R}_{+}^{2}\right)$ is nonconvex yielding $\overline{\text { cone }}\left(\operatorname{co~} f(C)-f(\bar{x})+\mathbb{R}_{+}^{2}\right)=\mathbb{R}^{2}$, by Proposition 5.5.

An analogous result is given by [13, Theorem 2.9] where cone $+(f(C)-f(\bar{x})+\operatorname{int} P)$ is considered instead: we note that by [13, Theorem 2.5], $\overline{\text { cone }}(f(C)-f(\bar{x})+P)$ is convex iff cone $+(f(C)-$ $f(\bar{x})+\operatorname{int} P)$ is convex, provided int $P \neq \emptyset$.

$$
\begin{aligned}
& \overline{\operatorname{cone}}\left(\mathcal{E}_{0 \bar{x}}\right)=\left\{\begin{array}{cc}
v=\beta u \\
v
\end{array}\right. \\
& {\left[S_{1}^{-}(\bar{x}) \cap S_{2}^{=}(\bar{x}) \neq \emptyset \text { and } S_{2}^{-}(\bar{x})=\emptyset\right] \text { or }} \\
& {\left[S_{1}^{-}(\bar{x}) \cap S_{2}^{+}(\bar{x}) \neq \emptyset, \beta=0 \text { and } S_{2}^{-}(\bar{x})=\emptyset\right]} \\
& {\left[S_{1}^{-}(\bar{x}) \cap S_{2}^{=}(\bar{x})=\emptyset, S_{1}^{-}(\bar{x}) \cap S_{2}^{+}(\bar{x}) \neq \emptyset, \beta<0\right. \text { and }} \\
& \left.S_{2}^{-}(\bar{x})=\emptyset\right] \\
& {\left[S_{1}^{-}(\bar{x})=\emptyset, S_{1}^{+}(\bar{x}) \cap S_{2}^{-}(\bar{x}) \neq \emptyset \text { and } \alpha>-\infty\right]}
\end{aligned}
$$




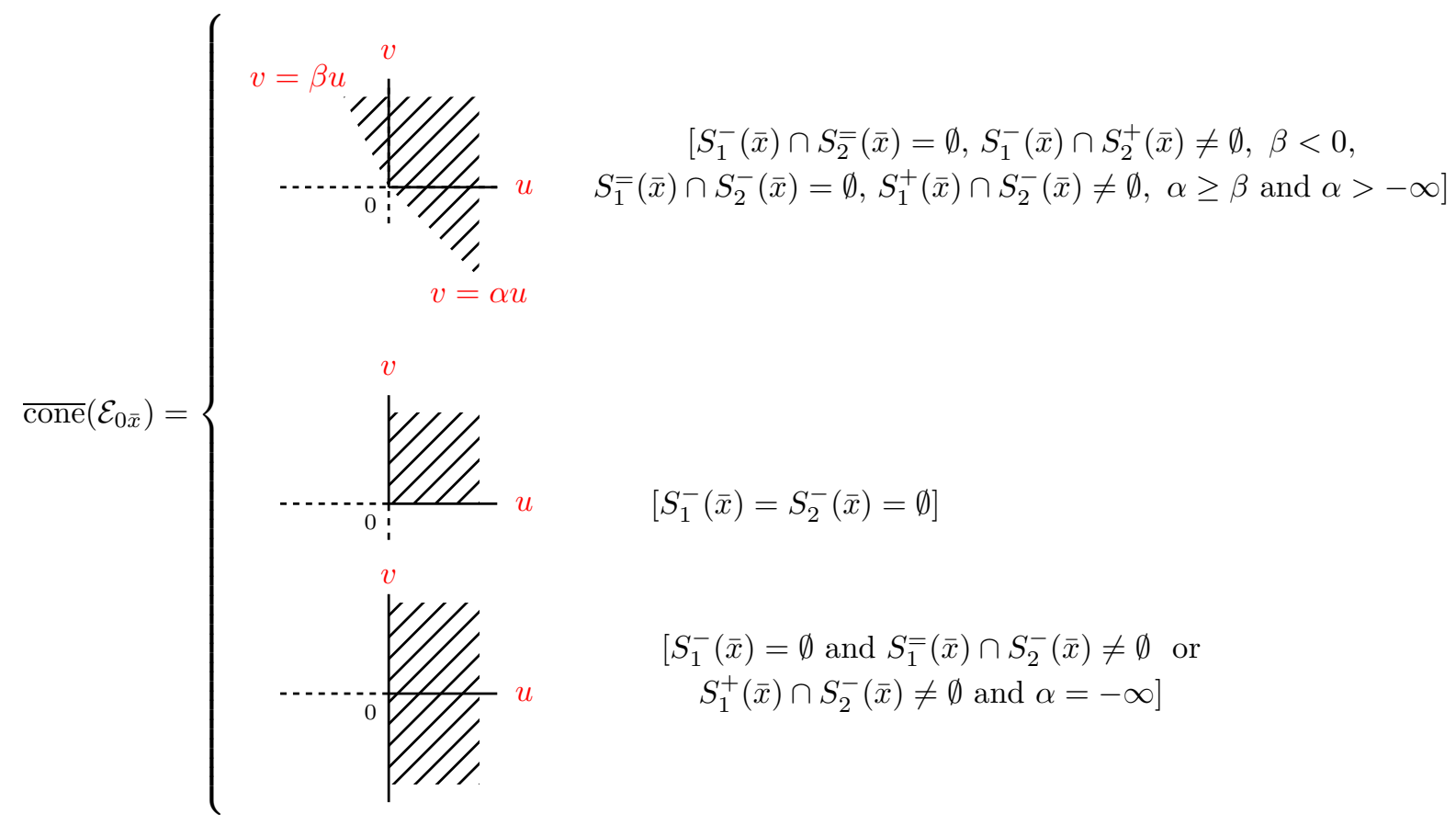

The following corollary is a direct consequence of the above description. We characterize the disjunction stated in Theorem 5.6 (b) (or, equivalently in Corollary 5.7) by means of the properties of the sets $S_{i}$ above defined.

Corollary 5.12. Assume that $P=\mathbb{R}_{+}^{2}$ and $\bar{x} \notin \operatorname{argmin}_{C} f_{i}, i=1,2$. The following assertions are equivalent :

(a) $\bar{x} \in \bigcup_{\left(p_{1}^{*}, p_{2}^{*}\right) \in \mathbb{R}_{++}^{2}}^{\operatorname{argmin}}\left(p_{1}^{*} f_{1}+p_{2}^{*} f_{2}\right)$;

(b) $\bar{x} \in E_{W}$ and $\left[S_{1}^{-}(\bar{x}) \cap S_{2}^{=}(\bar{x})=\emptyset, S_{1}^{-}(\bar{x}) \cap S_{2}^{+}(\bar{x}) \neq \emptyset, \beta<0, S_{1}^{=}(\bar{x}) \cap S_{2}^{-}(\bar{x})=\emptyset\right.$, $S_{1}^{+}(\bar{x}) \cap S_{2}^{-}(\bar{x}) \neq \emptyset, \alpha \geq \beta$ and $\left.\alpha>-\infty.\right]$

(c) $\bar{x} \in E$ and $\left[S_{1}^{-}(\bar{x}) \neq \emptyset, \beta<0, S_{2}^{-}(\bar{x}) \neq \emptyset, \alpha \geq \beta\right.$ and $\alpha>-\infty$. $]$

(d) $\bar{x} \in E_{p r}$ and $\left[S_{1}^{-}(\bar{x}) \neq \emptyset, \beta<0, S_{2}^{-}(\bar{x}) \neq \emptyset, \alpha \geq \beta\right.$ and $\left.\alpha>-\infty.\right]$

Proof. The equivalence between (a) and (b) follows from Corollary 5.7, where we set $S=$ int $\mathbb{R}_{+}^{2}$ or $S=\mathbb{R}_{+}^{2} \backslash\{(0,0)\}$, and the above description of the set $\overline{c o n e}\left(\right.$ co $\left.\mathcal{E}_{0 \bar{x}}\right)$, taking into account that $S_{i}^{-}(\bar{x})=\emptyset \Leftrightarrow \bar{x} \in \operatorname{argmin}_{C} f_{i}, i=1,2$.

As regards the equivalence between $(b)$ and $(c)$, it is enough to note that for $\bar{x} \in E$, we have:

$$
S_{i}^{-}(\bar{x}) \neq \emptyset \Leftrightarrow S_{i}^{-}(\bar{x}) \cap S_{j}^{+}(\bar{x}) \neq \emptyset, i \neq j .
$$

Concerning the equivalence between the previous assertions and $(d)$, we have already observed that (a) implies $\bar{x} \in E_{p r}$ (see Theorem 5.8), moreover (a) implies (c) so that (d) holds, while $(d)$ implies $(c)$ is straightforward. $\square$

\section{REFERENCES}


[1] H.P. Benson, An improved definition of proper efficiency for vector maximization with respect to cones, J. Math. Anal. Appl., 71 (1979), pp. 232-241.

[2] G. BIGI, Saddlepoint optimality criteria in vector optimization,in: Optimization in economics, finance and industry (Verona, 2001), Datanova, Milan, 2002, pp. 85-102.

[3] J. BonweIn, Proper efficient points for maximizations with respect to cones, SIAM J. Control and Optimization, 15 (1977), pp. 57-63.

[4] J.M. Borwein And R. Goebel, Notions of relative interior in Banach spaces, J. Math. Sci., 115 (2003), pp. $2542-2553$.

[5] J.M.Bonwein And A.S. Lewis, Partially finite convex programming, part I: Quasi relative interiors and duality theory, Math. Program. A, 57 (1992), pp. 15-48.

[6] R.I. Bot, E.R. Csetnek and G. Wanka, Regularity conditions via quasi-relative interior in convex programming, SIAM J. Optim., 19 (2008), pp. 217-233.

[7] R.I. Bot, S.M. Grad and G. Wanka, Duality in vector optimization, Springer, Berlin, 2009.

[8] R.I. Bot, E.R. Csetnek And A. Moldovan, Revisiting some duality theorems via the quasirelative interior in convex optimization, J. Optim. Theory Appl., 139 (2008), pp. 67-84.

[9] W.W. Brecker and G. Kassay, A systematization of convexity concepts for sets and functions, J. Convex Analysis, 4 (1997), pp. 109-127.

[10] F. Cammaroto and B. Di Bella,Separation theorem based on the quasirelative interior in convex optimization, J. Optim. Theory Appl. 125 (2005), pp. 223-229.

[11] P. Daniele And S. Giuffrè, General infinite dimensional duality and applications to evolutionary networks equilibrium problems, Optim. Letters, 1 (2007), pp. 227-243.

[12] L.L. Dines, On the mapping of quadratic forms, Bull. Am. Math. Soc., 47 (1941), pp. 494-498.

[13] F. Flores-BazÁn, F. Flores-BazÁn and C. Vera, Gordan-type alternative theorems and vector optimization revisited, in: Recent developments in vector optimization, Q. H. Ansari and J. C. Yao, eds, Vector Optim., Springer, Berlin, 2012, pp. 29-59,.

[14] F. Flores-BazÁn and E. Hernández, A unified vector optimization problem: complete scalarizations and applications, Optimization, 60 (2011), pp. 1399-1419.

[15] F. Flores-BAZÁn And E. HernÁNDEZ, Optimality conditions for a unified vector optimization problem with not necessarily preordering relations, J. Glob. Optim., 56 (2013), pp.299-315.

[16] F. Flores-Bazán and G. Mastroeni, Strong duality in cone constrained nonconvex optimization, SIAM J. Optim., 23 (2013), pp. 153-169.

[17] F. Flores-BazÁn And F. Opazo, Characterizing the convexity of joint-range for a pair of inhomogeneous quadratic functions and strong duality, Minimax Theory and its Applications, 1 (2016), pp. 257-290.

[18] F. Giannessi, Constrained Optimization and Image Space Analysis, Springer, 2005.

[19] A. GRAD, Quasi-relative interior-type constraint qualifications ensuring strong Lagrange duality for optimization problems with cone and affine constraints, J. Math. Anal. Appl., 361 (2010), pp. 86-95.

[20] C. Gutiérrez, L. Huerga And V. Novo, Scalarization and saddle points of approximate proper solutions in nearly subconvexlike vector optimization problems, J. Math. Anal. Appl. 389 (2012), pp. 1046-1058.

[21] S.M. GuU AND J. LI, Vector quasi-equilibrium problems: separation, saddle points and error bounds for the solution set, J. Glob. Optim., 58 (2014), pp. 751-767.

[22] B.Hernández-Jiménez, R. Osuna-Gomez and M.A. Rojas-Medar, Characterization of weakly efficient solutions for nonlinear multiobjective programming problems. Duality, J. Convex Anal., 21 (2014), pp. 1007-1022.

[23] G. J. O. Jameson, Ordered Linear Spaces, Lecture Notes in Math., 141, Springer -Verlag, Heidelberg, 1970.

[24] J. JAhn, Vector Optimization - Theory, Applications and extensions, Springer Verlag, Berlin, 2004.

[25] V.L. KLeE, Separation properties of convex cones, Proc. Amer. Math. Soc., 6 (1955), pp. 313-318.

[26] J. Li and G. Mastroeni, Image convexity of generalized systems and applications, J. Optim. Theory Appl., (2016), pp. 91-115.

[27] M.A. Limber ANd R.K. Goodrich, Quasi interiors, Lagrange multipliers, and $L^{p}$ spectral estimation with lattice bounds, J. Optim. Theory Appl., 78 (1993), pp. 143-161.

[28] D.T. Luc, Theory of Vector Optimization, Lecture Notes in Economics and Mathematical Systems, 319, 
Springer-Verlag, New York, Berlin, 1989.

[29] G. Mastroeni, Optimality conditions and image space analysis for vector optimization problems. In: Ansari Q H and Yao J-C, editors. Recent Developments in Vector Optimization, Berlin, Heidelberg: Springer-Verlag, 2012, pp. 169-220.

[30] F. TARDELLA, On the image of a constrained extremum problem and some applications to the existence of a minimum, J. Optim. Theory Appl., 60 (1989), pp. 93-104.

[31] R. T. Rockafellar, Convex Analysis, Princeton University Press, Princeton, 1970.

[32] C. ZăLinescu, Convex Analysis in General Vector Spaces, World Scientific, Singapore, 2002.

[33] C. ZăLInescu, On the use of quasi-relative interior in optimization, Optimization, 64 (2015), pp. 17951823.

[34] R. Zeng and R.J. Caron, Generalized Motzkin Gordan alternative theorems and vector optimization problems, J. Optim. Theory Appl., 131 (2006), pp. 281-289.

[35] Z.A. Zhou AND X.M. YANG, Optimality conditions of generalized subconvexlike set-valued optimization problems based on the quasi-relative interior, J. Optim. Theory Appl., 150 (2011), pp. 327-340. 